\title{
On the correlation between a sub-level qualifier refining the danger level with observations and models relating to the contributing factors of avalanche danger
}

\author{
Frank Techel $^{1}$, Stephanie Mayer ${ }^{1}$, Cristina Pérez-Guillén ${ }^{1}$, Günter Schmudlach ${ }^{2}$, and Kurt Winkler ${ }^{1}$ \\ ${ }^{1}$ WSL Institute for Snow and Avalanche Research SLF, Davos, Switzerland \\ ${ }^{2}$ Skitourenguru GmbH, Zurich, Switzerland
}

Correspondence: Frank Techel (techel@ slf.ch)

\begin{abstract}
Forecasting avalanche danger at a regional scale is a largely data-driven, yet also experience-based decision-making process. In the case of public avalanche forecasts, this process terminates in an expert judgment concerning summarizing avalanche conditions by using one of five danger levels. This strong simplification of the continuous, multi-dimensional nature of avalanche hazard allows for efficient communication but inevitably leads to a loss of information. Intending to overcome the discrepancy between determining the final target output in higher resolution while maintaining the well-established standard of assessing and communicating avalanche hazard using the avalanche danger scale, avalanche forecasters at the national avalanche warning service in Switzerland used an approach that combines absolute and relative judgments. First, forecasters make an absolute judgment using the five-level danger scale. In a second step, a relative judgment is made by specifying a sub-level describing the avalanche conditions relative to the chosen danger level. This approach takes into account the human ability to reliably estimate only a certain number of classes. Here, we analyze these (yet unpublished) sub-levels, comparing them with data representing the three contributing factors of avalanche hazard, snowpack stability, the frequency distribution of snowpack stability, and avalanche size. We analyze both data used in operational avalanche forecasting and data independent of the forecast, going back five years. Using a sequential analysis, we first establish which data is suitable and in which part of the danger scale by comparing their distributions at consecutive danger levels. In a second step, integrating these findings, we compare the frequency of locations with poor snow stability and the number and size of avalanches with the forecast sub-level. Overall, we find good agreement: a higher sub-level is generally related to more locations with poor snow stability and more avalanches of larger size. These results suggest that on average avalanche forecasters can make avalanche danger assessments with higher resolution than the five-level danger scale. Our findings are specific to the current forecast set up in Switzerland. However, we believe that avalanche warning services making a hazard assessment using a similar temporal and spatial scale as currently used in Switzerland should also be able to refine their assessments if (1) relevant data is sufficiently available in time and space, and (2) if a similar approach combining absolute and relative judgment is used. The sub-levels increase the predictive value of the forecast, opening the discussion on how this information could be provided to forecast users.
\end{abstract}




\section{Introduction}

In many snow-covered mountain regions, avalanche forecasts are disseminated to the public to inform and warn about avalanche conditions. The provision of these warnings to the public consists of two steps: first, a prediction of the avalanche hazard is made, and, second, the prediction is communicated in a forecast product.

Assessing and forecasting avalanche hazard is a largely empirical process in which a human forecaster analyzes and interprets data to make an informed judgment regarding current or expected avalanche conditions (e.g. LaChapelle, 1980; McClung, 2002; Floyer et al., 2016). During the hazard assessment process the four questions, What is the avalanche problem?, Where and when does it exist?, How likely is it that an avalanche will occur? and How big will the avalanche be? must be answered (Statham et al., 2018a). This requires assessing the three factors contributing to avalanche hazard (Fig. 1a; Techel et al., 2020a; EAWS, 2021):

- Snowpack stability describes the stability of the snowpack at a point (Techel et al., 2020a). Snowpack stability is inversely related to the probability of avalanche release. It is also referred to as the sensitivity to triggers (CMAH, Statham et al., 2018a), which assesses the sensitivity of the snowpack to fail given a specific triggering level (Statham et al., 2018a), as for instance a person skiing a slope.

- The frequency distribution of snowpack stability describes the respective proportions of spots where triggering an avalanche given a specific triggering level is possible (Techel et al., 2020a; EAWS, 2021). It is also referred to as the spatial distribution (Statham et al., 2018a). The sensitivity to triggers and the spatial distribution describe the likelihood of avalanches in the CMAH.

- Avalanche size refers to the destructive potential of avalanches.

Once all relevant avalanche problems have been identified, their location and temporal occurrence specified, and their character described, avalanche hazard is summarized in regional avalanche forecasts using one of five danger levels (cf. Fig. 1b) according to a danger scale (i.e. in Europe the European Avalanche Danger Scale, EADS; EAWS, 2020). Aspects and elevation range where the danger and/or where the avalanche problems prevail are highlighted in the forecast products. Hence, a human forecaster reduces the avalanche conditions, continuous and multi-dimensional in nature, to a set of symbols (levels, classes, terms, text) representing this reality (LaChapelle, 1980; Hutter et al., 2021). As pointed out by Murphy (1993), the description of a continuous phenomenon using a discrete number (or level) inevitably leads to a loss of information. In addition, a coarse resolution may lead to considerable differences within a (spatial or temporal) unit or a class (e.g. within a danger level; SLF, 2020). It is therefore important that avalanche forecasters assess avalanche danger as detailed as possible when preparing a public forecast, given the available data and resources. This level of detail may be greater than what is communicated in the forecast product (e.g. Walcher et al., 2018; Techel et al., 2020b).

An increased level of detail may include, for instance, decomposing the judgmental forecasting process and specifying each of the individual components relevant for the final hazard assessment (MacGregor, 2001; Statham et al., 2018a). It may, however, also entail to actually increase the resolution of the hazard assessment either in a spatial or temporal context, or with 
(a) Contributing factors of avalanche hazard

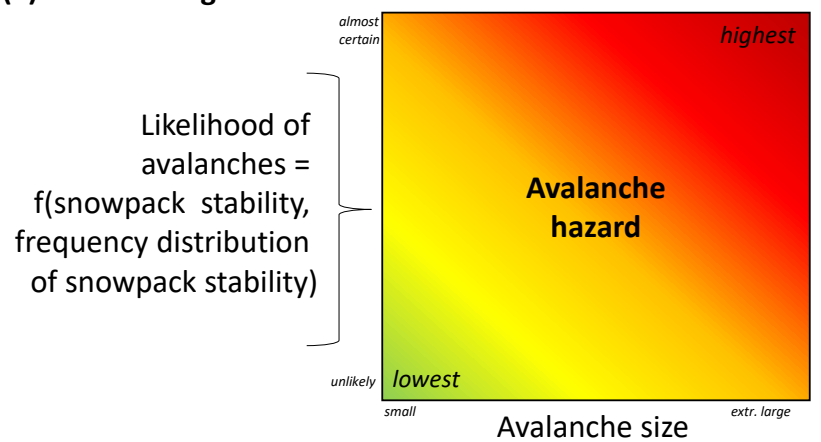

(b) Danger levels

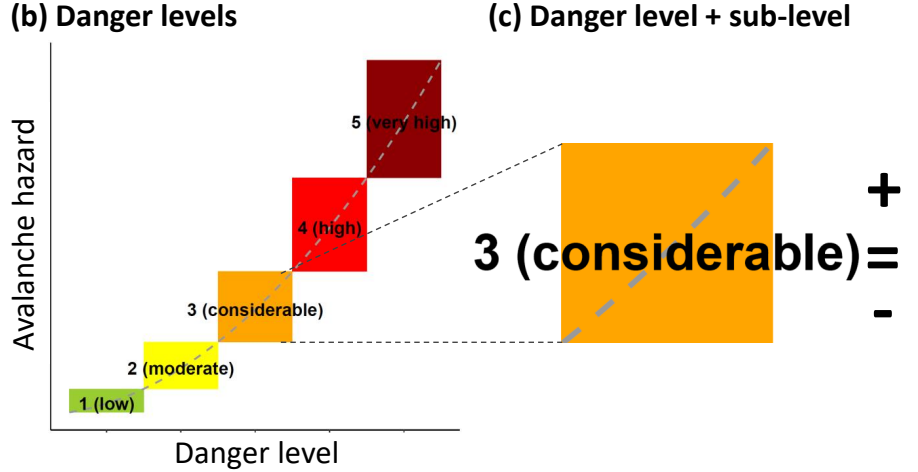

Figure 1. (a) Avalanche hazard chart. The contributing factors of avalanche hazard are snowpack stability, the frequency distribution of snowpack stability, and avalanche size (Techel et al., 2020a; EAWS, 2021). In the CMAH, these are termed the likelihood of triggering and the destructive avalanche size (Statham et al., 2018a). (b) Avalanche hazard, continuous in Nature, is summarized using five ordinal danger levels. (c) In Switzerland, three ordinal sub-levels are assigned to danger levels indicating whether the hazard is high (+ or plus), in the middle (= or neutral), or low (- or minus) within a respective level. The gradient of the colour transition (a) and the shape of the curve (b) are for illustration purpose only.

regard to assessing the individual components of avalanche hazard, or of avalanche danger itself. Increasing the temporal and spatial resolution primarily requires a sufficient amount of relevant data in time and space, and the resources to efficiently analyze these data. In contrast, increasing the resolution of the danger scale to a resolution greater than the existing five levels requires clear definitions of these levels. Furthermore, making judgments on a scale with many options contrasts with the wellestablished finding that absolute judgments on a scale with more than seven points becomes unreliable (Miller, 1956). However, alternatively, a two-step approach can be used, which combines absolute and comparative judgments (Goffin and Olson, 2011; Kahneman et al., 2021): Following such an approach, a first assessment is made using a small number of categories relying on guidelines or definitions. In the case of avalanche forecasting, this could be the step to assign a danger level according to the five-level avalanche danger scale (EAWS, 2018). In a second step, a relative rating is made with regard to this level (Kahneman et al., 2021). Compared to absolute judgments, this approach requires more effort and is time-consuming, but allows a finer discrimination within previously assigned categories (Kahneman et al., 2021). Such an approach has been used during the past five years in Switzerland, where forecasters assigned a danger level and a sub-level qualifier refining where within this danger level the avalanche conditions are expected (Techel et al., 2020b). This leads to our over-arching research question: using such an approach to assign a sub-level qualifier to a danger level, can human avalanche forecasters forecast avalanche hazard at finer granularity than the five danger levels?

Unfortunately, addressing this question is not straightforward as avalanche danger and, hence, the sub-levels cannot be measured. However, as the danger levels represent a rank order in terms of the severity of the avalanche conditions - with snow stability decreasing, the number of potential triggering locations and of avalanche size increasing with increasing danger level (EAWS, 2018; Techel et al., 2020a, e.g.), we tackle this question using a comparative approach: we test whether there is 
a positive monotonic correlation between the sub-levels assigned to danger levels and data describing the three contributing factors of avalanche hazard. We therefore do not ask: is the forecast danger level or sub-level correct? but merely: is there a rank order relationship between the data and the sub-levels? For this, we make use of both observational data collected for the purpose of avalanche forecasting in Switzerland as well as independent data sources not used in the forecasting process: the output from two recently developed models (Pérez-Guillén et al., 2021; Mayer et al., 2022), and to data related to avalanche risk (Winkler et al., 2021).

We first determine for each parameter in what range of the danger scale it correlates with the forecast danger levels $(D)$. Here, we assume that the forecast danger level is correct on average, which has been shown for Switzerland (e.g. Techel and Schweizer, 2017; Schweizer et al., 2021) but also for other forecasts (e.g. Logan and Greene, 2018; Statham et al., 2018b). If a correlation exists, and given that the sub-levels ( $D_{\text {sub }}$ ) are used consistently, we can expect a correlation between the sub-levels ( $\left.D_{\text {sub }}\right)$ and the data as well. Therefore, in this study, we ask the following two questions:

1. Does a data source representing a contributing factor of avalanche hazard correlate with the danger level $D$ ? If so, in which range of the danger scale?

2. For the range in the danger scale determined in (1), is there a monotonically increasing correlation between the parameter representing a contributing factor and the sub-levels $D_{\text {sub }}$ as well?

\section{Avalanche forecast in Switzerland - brief overview and approach to assign a sub-level qualifier to the danger level}

The Swiss avalanche forecast has previously been described in several publications (Techel and Schweizer, 2017; SLF, 2020; Hutter et al., 2021). Here, we therefore only summarize some key facts.

During winter, the national avalanche warning service at the WSL Institute for Snow and Avalanche Research SLF (SLF) publishes an avalanche forecast at 17.00 CET, valid until 17.00 CET the following day (see example in Figure 2a). This forecast is updated at 08.00 CET during the main winter season. Definitions and guidelines provided by the European Avalanche Warning Services (EAWS) are used when assessing and communicating avalanche danger. A team of eight forecasters is involved in the production of the forecasts.

The production of the forecast always starts with the assessment of the current avalanche conditions. Numerous data are used in this process. These include measurements from automated weather stations located at the elevation of potential avalanche starting zones (SLF, 2022), simulations from the physical snow-cover model SNOWPACK (Lehning et al., 2002) driven with these measurements, and observational data collected for the purpose of avalanche forecasting. For the actual forecast, forecasters primarily use the numerical weather prediction model COSMO with $1 \mathrm{~km}$ resolution (MeteoSwiss, 2022). The three forecasters together on duty individually draw up their hazard assessment for the entire forecast domain. In a group discussion at the forecaster briefing, these assessments are combined resulting in one consolidated forecast for the following 24-hour forecast period.

The Swiss avalanche forecast describes regional avalanche conditions. The average size of the almost 150 warning regions, the smallest spatial units used in the forecast, is about $200 \mathrm{~km}^{2}$ (white polygons in Fig. 2b). However, depending on conditions, 
(a)

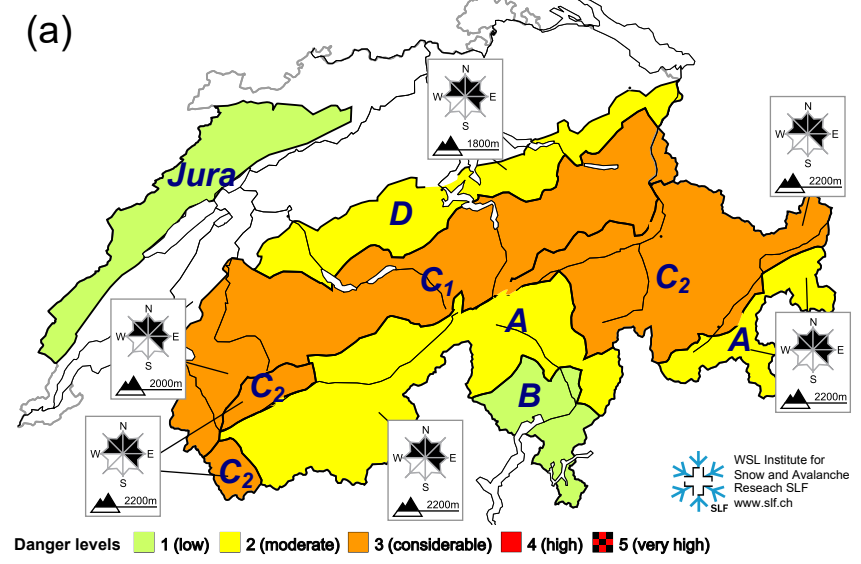

(b)

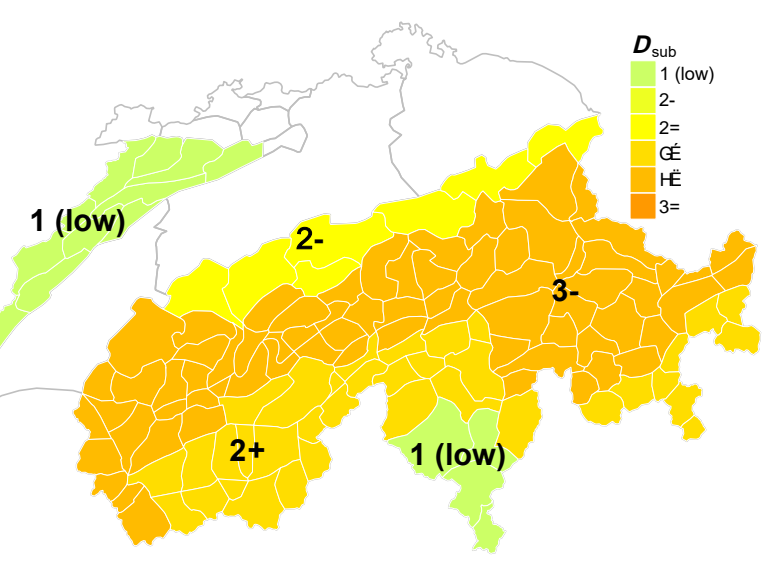

Figure 2. Maps of Switzerland showing (a) the avalanche forecast published on 10 March 2018 and (b) the (unpublished) sub-levels for this forecast. In addition, in (b) the warning regions (white polygon boundaries), the smallest spatial units used in the Swiss forecast, are shown. These are aggregated to danger regions in the published forecast (i.e. region C1 in a). Source: example is taken from Techel et al. (2020b).

these warning regions are flexibly aggregated to danger regions (i.e. region A in in Fig. 2a), where avalanche conditions are considered similar and are described with the same danger level, critical aspects and elevations where the danger prevails, avalanche problems and danger description. In addition, since the winter of 2016/2017 forecasters assess where within a danger level the avalanche conditions are expected. To do so, an approach combining absolute and comparative judgements, as described in the previous section, is used. Forecasters first assign a danger level according to the definitions in the EADS, and then make a comparative refinement using one of three qualifier terms (Techel et al., 2020b):

- plus or + : the danger is assessed as high within the level, e.g. a $3^{+}$is high within 3 (considerable) ${ }^{1}$

- neutral or $=$ : the danger is assessed as about in the middle of the level, e.g. a $3^{=}$is about in the middle of 3 (considerable)

- minus or -: the danger is assessed as low within the level, e.g. a $3^{-}$is low within 3 (considerable)

It is of note that the criteria, which should be applied, and the range covered by a sub-level within a danger level, remained undefined. Furthermore, forecasters made no such differentiation for 1 (low), as a further distinction within this level seemed impossible. In addition, an internal analysis of qualifiers assigned to danger levels describing wet-snow conditions showed that forecasters used the sub-level qualifiers inconsistently. Hence, for wet-snow conditions, the assignment of sub-level qualifiers was halted after a test winter. 
Table 1. Overview showing the analyzed data sources and the contributing factors of avalanche hazard (snowpack stability, the frequency of snowpack stability, avalanche size) for which we consider the respective data sources to be a proxy. $x$ refers to a contributing factor which was analyzed, $(x)$ to a factor, which is included in the variable but does not vary (i.e. for natural avalanches the stability class (type of trigger) is constant $=$ natural release ).

\begin{tabular}{|c|c|c|c|c|c|c|}
\hline \multicolumn{2}{|l|}{ data source } & stability & frequency & size & $D$ & $N$ \\
\hline \multirow[t]{3}{*}{ observations } & natural avalanches & $(\mathrm{x})$ & $\mathrm{x}$ & $\mathrm{x}$ & & 8956 avalanches \\
\hline & human-triggered avalanches & $(\mathrm{x})$ & $\mathrm{x}$ & $\mathrm{x}$ & & 1814 avalanches \\
\hline & human-triggered whumpfs & $(\mathrm{x})$ & $\mathrm{x}$ & & & 5996 observations \\
\hline \multirow[t]{2}{*}{ stability tests } & Rutschblock test & $\mathrm{x}$ & $\mathrm{x}$ & & & 2201 tests \\
\hline & Extended Column Test & $\mathrm{x}$ & $\mathrm{x}$ & & & 2461 tests \\
\hline \multicolumn{2}{|c|}{ accident and movement points } & $(\mathrm{x})$ & $\mathrm{x}$ & & & 379 accidents, 976087 movement points \\
\hline \multirow[t]{2}{*}{ models } & danger level & & & & $\mathrm{x}$ & 452 days for up to 122 stations \\
\hline & instability & $\mathrm{x}$ & $\mathrm{x}$ & & & 725 days for up to 124 stations \\
\hline
\end{tabular}

\section{Data description and preparation}

We analyzed observational data which was collected as part of operational avalanche forecasting (Sect. 3.2). As far as observations were available at the time when forecasters produced the forecast for the following day, they were considered in the assessment of the current avalanche conditions. Moreover, we also used external data and two recently developed models which were not available during the forecast process (Sect.s 3.3 and 3.4). Data from five winters 2016/2017 to 2020/2021 were used; for the danger-level model (Sect. 3.4.1) only data from winters 2018/2019 to 2020/2021 were available.

In the following, we describe the data and their preparation for this analysis.

\subsection{Avalanche forecast}

We extracted the forecast danger level, the unpublished sub-level, and the critical aspects and elevations, referred to as the core zone (Fig. 2a and b) which described dry-snow conditions in the Swiss Alps. We used the forecasts issued at 17.00 CET, valid until the following day at $17.00 \mathrm{CET}$. These forecasts were published on 832 days.

\subsection{Observations}

\subsubsection{Avalanche observations}

The occurrence of avalanches directly indicates instability (e.g McClung and Schaerer, 2006). Avalanche occurrence data can provide information on all three contributing factors (Tab. 1), snow instability (i.e. an avalanche released naturally), the frequency of unstable locations (i.e. the number of naturally-released avalanches), and avalanche size (Schweizer et al., 2020).

\footnotetext{
${ }^{1}$ we refer to the danger levels by integer-signal word (i.e. 3 (considerable)), and to the sub-levels by the integer-qualifier (i.e. $3^{+}$)
} 
In Switzerland, about 80 «stationary» observers report avalanches in their region on a daily basis. Observers report avalanches either individually or by aggregating avalanches into an avalanche summary report. In addition to avalanches regularly reported by these observers, field observers, which are also part of the observer network, and the public may report avalanches. Reported avalanche properties include the location and the estimated time of the release, the avalanche size (size classes 1 to 5 according to EAWS (2019)), the moisture content (dry or wet) and the trigger type (i.e. natural release, human-triggered; SLF, 2020). Observers also indicate when there was no avalanche.

Natural avalanches: We extracted all avalanches of size 2 or larger, with trigger type natural release. We excluded avalanches classified as a wet-snow or gliding avalanche. To increase the overall quality of the observations with regard to consistent reporting of avalanche activity (or absence of activity), we reduced the data set considering only the $20 \%$ of the warning regions with the highest number of days with at least one dry-snow avalanche. These warning regions are marked in Appendix Figure A1a. In total, 8956 avalanches fulfilled these criteria. In addition, observers reported no avalanches in 8826 cases.

Human-triggered avalanches: For human-triggered avalanches, of which a large share is reported by rescue services and the public, we considered reported events when the trigger type was human-triggered, and the avalanche size was size 2 or larger or when a person was caught in the avalanche, and which were not classified as a wet-snow or gliding avalanche. For the purpose of this analysis, we assigned size class 2, if a size estimate was missing, which was the case for 151 of the 603 accidental avalanches but also for the 23 accidental avalanches classified as size 1. In total, 1814 human-triggered avalanches were considered in this analysis (their spatial distribution is shown in the Appendix Fig. A1b). These were triggered during backcountry touring (i.e. during a ski- or snowshoe-tour) or during riding in unsecured avalanche terrain close to ski areas.

\subsubsection{Human-triggered whumpfs and shooting cracks}

Whumpfs, a sudden, collapse-type failure of a weak layer due to rapid localized loading (Schweizer and Jamieson, 2010) - as for instance by a human, and shooting cracks in the snowpack provide an indication of the presence of locations potentially prone to triggering by a human (Tab. 1).

When reporting their observations after a day in the field, observers also report whether they observed human-triggered whumpfs and shooting cracks, and how frequent these danger signs occurred using three classes (DS.class) none ( 0 such observations), rare (1 to 3 such observations) and frequent ( $>3$ observations; SLF, 2020).

We extracted all observations, which were reported after a day in the field. This resulted in 5996 observations.

\subsubsection{Stability tests}

Information on snow stability can also be obtained by digging a snow pit and performing a stability test. These tests primarily provide a very localized information of snow stability. Therefore, to obtain information on the frequency distribution of snowpack stability, numerous tests must be performed on the same day and in the same region (e.g. Birkeland, 2001; Schweizer et al., 2003). Alternatively, tests obtained under similar avalanche conditions may be combined to derive typical stability distributions 


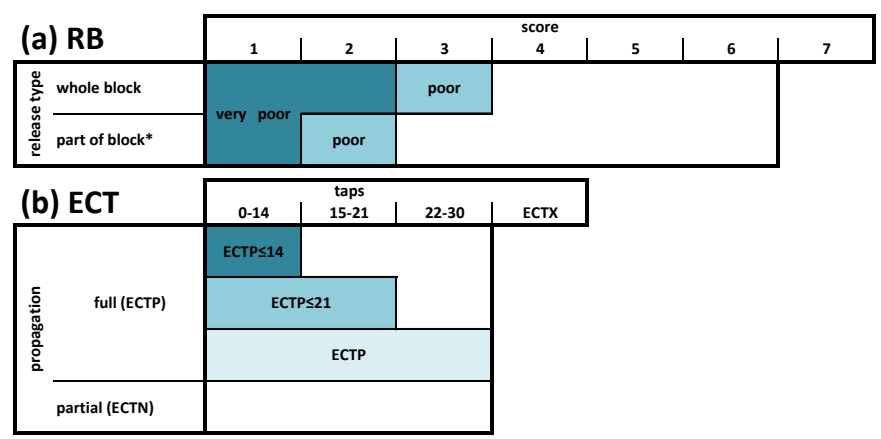

Figure 3. Classification of stability tests (a) Rutschblock (RB) and (b) Extended Column Test (ECT). The RB classification (RB.class) considers the score (7 loading steps) and the release type (whole block, part of block and edge only*). Similarly, ECT are classified combining the number of taps to initiate a fracture (30 loading steps) and the propagation propensity (full propagation: ECTP, partial or no propagation: ECTN). RB score 7 and ECTX indicate that no failure could be initiated following loading.

In Switzerland, two stability tests are performed regularly by observers to assess snowpack stability, the Rutschblock test (RB; Schweizer, 2002; SLF, 2020) and the Extended Column Test (ECT; Simenhois and Birkeland, 2009; SLF, 2020). With these tests, the stability of an isolated block of snow is tested by loading the block according to the defined loading steps by a human (RB), or by tapping with the hand on a shovel blade lying on top of the snow column (ECT) until a fracture in the column is observed. The interpretation of the test results considers the type of release (i.e. fracture across the entire block, or only part of the block) and the loading step. For an overview and comparison of the two tests refer to Techel et al. (2020c).

Rutschblock (RB): We classified the RB results according to the classification by Techel et al. (2020a) into four stability classes (RB.class: very poor, poor, fair, good). However, in this analysis, we considered exclusively the two classes very poor and poor (Fig. 3a), as these are most closely linked to unstable conditions (Schweizer and Jamieson, 2010; Techel et al., 2020c).

Extended Column Test (ECT): We treated a test result as potentially unstable if a fracture propagated within one tap across the whole column (ECTP; Winkler and Schweizer, 2009). In addition, fracture propensity was combined with three different fracture initiation criteria as suggested in previous studies (Simenhois and Birkeland, 2009; Winkler and Schweizer, 2009; Techel et al., 2020c). The corresponding three stability classes are shown in Figure $3 \mathrm{~b}$.

In total, 2201 RB and 2261 ECT were available. Their spatial distribution is shown in Fig. A1c in the Appendix.

\subsection{Accidental avalanches and backcountry touring activity}

Recently, Winkler et al. (2021) analyzed avalanche risk during backcountry touring in Switzerland. In their analysis, Winkler et al. relied on a data set of accident points extracted from the accident data base at SLF, and movement points in potential avalanche terrain extracted from GPS tracks recorded during backcountry ski tours in Switzerland (Schmudlach, 2021). Avalanche risk, as defined by Winkler et al., is the ratio of events (accident points) to events and non-events (accident and movement points combined) after backcountry users have adapted their behaviour to the conditions. This ratio is closely re- 
lated to the density of locations where triggering of an accidental avalanche by a human is possible, and, thus, in a more general way also to the density of potential triggering locations (Tab. 1).

We relied on an updated version of the data-set used by Winkler et al. (2021), including the two most recent winters 2019/2020 and 2020/2021. We filtered the data according to the specification by Winkler et al. (2021) keeping points located in potential avalanche terrain. In total, the data set contains 379 avalanche accident points and 976087 movement points extracted from 2'519 individual GPS tracks.

\subsection{Models (random forest classifiers) based on snow-cover simulations}

In addition to observational data, we analyzed the output of two recently developed random forest classifiers predicting the danger level (Pérez-Guillén et al., 2021) or snow-cover instability (Mayer et al., 2022). Both models use snow-cover simulations from the operational SNOWPACK model (Lehning et al., 2002) driven with data from 124 automatic weather stations as input (Lehning et al., 1999; Morin et al., 2019). An overview of the spatial distribution of these stations is provided in the Appendix Figure A1d. These stations are situated at the elevation of potential avalanche starting zones. In addition to simulations for flat study plots, snow-cover simulations are operationally made for virtual slopes with a slope incline of $38^{\circ}$ and the four slope orientations N, E, S, W (Morin et al., 2019). During the explored winter seasons, these two random forest models were not used during the forecast production process.

\subsubsection{Danger-level model}

The first model, which we refer to as the danger-level model, was trained with a large data set of quality-checked danger levels spanning more than 20 years (Pérez-Guillén et al., 2021). The random forest classifier (Breiman, 2001) uses 30 features, describing both measured meteorological conditions (24-hour averaged values) and snow-cover properties simulated with the SNOWPACK model. The random forecast classifier provides the probabilities ( $p r o b$ ) for the four danger levels 1 (low) to 4 (high) relying on an ensemble of 1000 classification trees. We used the model predictions relying on daily average weather variables and features extracted from the simulated snow stratigraphy at $12.00 \mathrm{CET}$ on the day of interest. In total, model output was available for 452 days and at 122 stations for simulations made for the four virtual slope orientations $\mathrm{N}, \mathrm{E}, \mathrm{S}, \mathrm{W}$.

\subsubsection{Instability model}

The second model developed by Mayer et al. (2022) - we refer to it as the instability model - also uses snow-cover simulations provided by the SNOWPACK model to assess snow instability. The instability model uses six variables describing the potential weak layer and the overlying slab to predict the probability prob $_{\text {unstab }}$ that a snow layer is unstable. Based on an ensemble of 400 classification trees, the output probability ranges from 0 (a layer was classified as stable by all the trees) to 1 (all trees classified it as unstable). We used the simulated snow stratigraphy at 12.00 CET on the day of interest, considering the simulations for the virtual slopes as for the danger-level model. Model output was available on 725 days and for up to 124 automatic weather stations. 


\begin{tabular}{|c|c|c|}
\hline Workflow & (a) & (b) \\
\hline $\begin{array}{l}\text { (1) Number of data points } \\
\text { per danger region? }\end{array}$ & $\begin{array}{l}\text { often only } 1 \\
\text { (or none) }\end{array}$ & many \\
\hline $\begin{array}{l}\text { (2) Calculate metric } \\
\text { per danger region }\end{array}$ & & $\begin{array}{l}\text { - } \text { natural avalanches } \rho_{\text {nat,i }}, A A I \\
\text { - } \text { human-triggered avalanches } \rho_{\text {hum }, \mathrm{i}} \\
\text { - } \text { danger-level model* } D_{\text {model }} \\
\text { - instability model* } P_{\text {unstab }}\end{array}$ \\
\hline \multicolumn{3}{|c|}{ (3) Aggregate data points for each $D$ or $D_{\text {sub }}$} \\
\hline \multicolumn{3}{|c|}{ (4) Test subsets of data to answer RQ1 } \\
\hline $\begin{array}{l}\text { (5) Resample, calculate } \\
\text { median and confidence } \\
\text { interval for each } D \text { or } D_{\text {sub }} \\
\text { * plus core-zone specific statistic }\end{array}$ & $\begin{array}{ll}- & \text { Danger signs } \tilde{P}_{\text {DS.class }} \\
\text { - } & \text { Rutschblock test } \widetilde{P}_{\text {RB.class }} \\
\text { - } & \text { Extended Column Test } \tilde{P}_{\text {ECT.class }} \\
\text { - } & \text { acc.-move. points* } \tilde{R}_{\text {acc/move }}\end{array}$ & $\begin{array}{lll}- & \text { natural avalanches } \tilde{\rho}_{\text {nat,i }} \quad \widetilde{A A} I \\
\text { - } & \text { human-triggered avalanches } & \tilde{\rho}_{\text {hum,i }} \\
\text { - } & \text { danger-level model* } \widetilde{D}_{\text {model }} \\
\text { - } & \text { instability model* } \tilde{P}_{\text {unstab }}\end{array}$ \\
\hline (6) Compare subsets of dat & answer RQ2 & \\
\hline
\end{tabular}

Figure 4. Workflow: preparatory steps (steps 1 to 3) and analysis to answer research question 1 (step 4) and 2 (steps 5 and 6 ).

\section{Methods}

\subsection{Definition of parameters}

We linked the forecast with the observations and the model output by their location and calendar day.

For this analysis, we distinguished between data sources which mostly included only a single data point (or even no data) was available for a forecast danger region (Fig. $4 \mathrm{a}$ - step 1) and data which allowed the calculation of a proportion or a mean for each forecast danger region (Fig. 4b). The first group included observations of danger signs, stability test results, and the accident and movement points, while the second group contained observations of natural and human-triggered avalanches and the predictions of the two models.

For the data sources with sufficient data points per danger region, we defined the following parameters that summarize the observations or modelled output for a given danger region (i.e. for the danger region A in Fig. 2a). This step is shown as step 2 in Fig. 4b.

Natural avalanches: We derived a metric describing the spatial density of natural avalanche occurrence ( $\left.\rho_{\text {nat, } i}\right)$ within a danger region. This metric expresses the number of reported natural avalanches $\left(N_{\text {nat,i }}\right)$ equal or greater a certain size class 
$i:=\{2,3, \geq 4\}$ relative to the surface area considered as potential avalanche release areas $\left(A_{\mathrm{PRA}}\right)$ in this danger region:

$\rho_{\text {nat,i }}=\frac{N_{\text {nat, } \mathrm{i}}}{A_{\text {PRA }}}$

We used the potential release area (PRA) delineation by Bühler et al. (2018, Fig. A1a). This automatic release area delineation relies on terrain characteristics, as for instance, elevation, slope angle, curvature and forestation, derived from a digital elevation model with $5 \mathrm{~m}$ resolution (Bühler et al., 2018).

Furthermore, for each danger region, we derived an avalanche activity index ( $A A I$ ) relative to $A_{\mathrm{PRA}}$. We defined the $A A I$ as sum of the natural avalanches weighted by their size with the weights $w_{\mathrm{i}}:=\{0.1,1,10\}$ for size classes $i:=\{2,3, \geq 4\}$, scaled with $A_{\text {PRA }}$ :

$A A I=\frac{\sum_{i=2}^{4} N_{\text {nat, } \mathrm{i}} w_{\mathrm{i}}}{A_{\mathrm{PRA}}}$

Human-triggered avalanches: Similar to natural avalanches, we defined the spatial density of human-triggered avalanches as:

$\rho_{\text {hum }, \mathrm{i}}=\frac{N_{\text {hum }, \mathrm{i}}}{A_{\text {PRA }}}$,

where $N_{\text {hum, }}$ is the number of human-triggered avalanches equal or greater than size $i:=\{\geq 2, \geq 3\}$.

Danger-level model: The model provides the danger-level predictions of 1000 individual classification trees. Following the definition for the expected value of a discrete random variable (Kuter, 2020), we derived a weighted mean danger rating $D$ for each automated weather station $(s t)$ and for each of the four virtual slope aspects (asp:= N, E, S, W) by incorporating the expected probability prob for a danger level $d$ (1 (low), 2 (moderate), 3 (considerable), 4 (high)):

$D_{\text {st,asp }}=\sum_{d=1}^{4} w_{\mathrm{d}} \operatorname{prob}(d)$.

where $w_{\mathrm{d}}$ is a numeric value assigned to a danger level $d$ and $\operatorname{prob}(d)$ the predicted class probability for each danger level $d$.

In a second step, for each danger region with the same forecast $D_{\text {sub }}$, we combined the $N$ predicted $D_{\text {st,asp }}$ to obtain a mean model-predicted danger rating:

$D_{\text {model }}=\frac{1}{N} \sum_{\text {st,asp }=1}^{N} D_{\text {st,asp }}$

Danger levels are rank-ordered, hence, the increase in danger from one danger level to the next is unknown. To derive the expected danger rating $D$, we used the respective integer values of the four danger levels 1 (low) to 4 (high) $(w:=\{1,2,3,4\}$ ). However, to address the uncertainty related to $w$, we tested $\left(w:=\left\{1, f, f^{2}, f^{3}\right\}\right)$ for various $f$, as for instance for $f=1.5$ or $f=5$. The resulting $D_{\text {model }}$ vary in absolute values, but are highly correlated (Pearson correlation coefficient $r=[0.91,0.99]$ ).

Instability model: Following the approach suggested by Mayer et al. (2022), we identified the layer with the highest $p r o b_{\text {unstab-value }}\left(\max \left(\right.\right.$ prob $\left.\left._{\text {unstab }}\right)\right)$ as potential weak layer within each simulated profile. Depending on the value of $\left.\max \left(\operatorname{prob}_{\text {unstab }}\right)\right)$, 
the profile was then classified as unstable or stable using the suggested threshold of $\max \left(\operatorname{prob}_{\text {unstab }}\right) \geq 0.77$. Similar to the danger-level model, we derived the proportion of profiles classified as unstable, $P_{\text {unstab}}$, for each danger region:

$P_{\text {unstab }}=\frac{N\left(\max \left(\text { prob }_{\mathrm{unstab}} \geq 0.77\right)\right)}{N}$,

where $N\left(\max \left(\operatorname{prob}_{\text {unstab }}\right) \geq 0.77\right)$ is the number of simulated profiles classified as unstable, and $N$ the number of simulated profiles.

Further parameters: In addition to these variables, we derived the following proportions and ratios combining all data points for a danger level, $d$, or sub-level, $s$ (step 3 in Fig. 4),:

- the proportion $P$ of observations or stability test results which fulfilled a certain criteria $\left(P_{\mathrm{DS} . c l a s s,}, P_{\mathrm{RB} . c l a s s}, P_{\mathrm{ECT} . c l a s s}\right)$, and

- the accident-movement point ratio $\left(R_{\text {acc/move }}\right)$ as in Winkler et al. (2021).

Not all the data sources describing the contributing factors are equally suitable to explore differences between all the danger levels or sub-levels in the entire range of the danger scale:

- The occurrence of natural avalanches of increasing size is a key criterion defining the higher danger levels in the avalanche danger scale (EAWS, 2018); therefore we analyzed the occurrence of natural avalanches for the entire danger scale despite the number of cases being comparably small due to the fact that higher danger levels (and thus $D_{\text {sub }}$ ) are much less frequently forecast.

- For data which relies on a human being present in avalanche terrain, we combined the (few) cases at 4 (high) and 5 (very high). At these danger levels, travel in avalanche terrain is strongly reduced due to dangerous conditions leading to a strong reduction in observational data.

- For each of the two models, we combined the predictions at 4 (high) and 5 (very high), as the models relied on training data either merging these two danger levels (danger-level model, Pérez-Guillén et al., 2021), or - in the case of the instability model - the few cases observed at 4 (high) were merged with 3 (considerable) (Mayer et al., 2022).

\subsection{Data analysis and presentation}

To answer research question 1 Does a data source representing a contributing factor correlate with the danger levels D? If it does, in which range of the danger scale?, we tested whether (a) values of a parameter $x$ referring to a given data source were significantly different between two neighbouring danger levels $D(d, d+1)$, and (b) whether values increased with increasing danger level. To do so, we applied either the Wilcoxon rank-sum test (Hollander and Wolfe, 1973, p. 68; R-function: wilcox.test) or a proportion test (Newcombe, 1998, R-function: prop.test) testing the data for the one-sided hypothesis whether (a) and (b) were fulfilled at the $p \leq 0.05$ level. This procedure was important, as it provided an indication of the range in the danger scale where the observations showed a monotonic increase with increasing $D$, and, hence, where such a trend should 


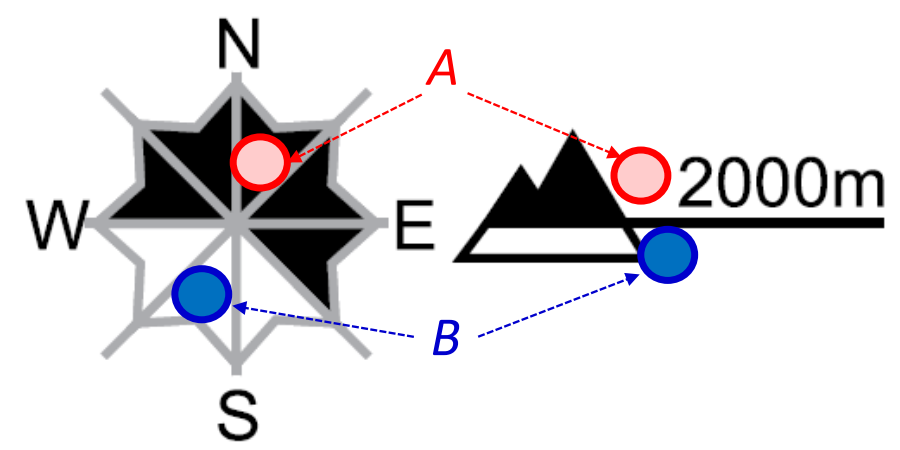

Figure 5. Graphical representation of the critical aspects (colored black in the aspect rose, here W - N - SE) and the critical threshold elevation (here $2000 \mathrm{~m}$ a.s.1.) indicated in the Swiss avalanche forecast. The points A and B are described in the text.

also be seen for $D_{\text {sub }}$ if the sub-levels were used consistently (research question 2: For this range in the danger scale, is there a monotonically increasing correlation between the parameter representing a contributing factor and the sub-levels $D_{\text {sub }}$ as well?). Moreover, we checked whether a monotonic, positive correlation between the metric of interest and $D_{\text {sub }}$ existed. To this end, we calculated the Spearman rank-order correlation coefficient $r_{\mathrm{s}}$ (Wilks, 2011, p. 55).

To obtain a better understanding of the distribution of the samples, we calculated the bootstrap-sampled median $\tilde{x}_{\mathrm{d}}$ and a 95\% confidence interval CI (Efron, 1979; Ramachandran and Tsokos, 2021). To do so, we randomly sampled 1000 times $N$ data points with replacement for each $d_{\mathrm{i}}$, where $N$ is the number of samples for a respective $d$. The $95 \% \mathrm{CI}$ is defined as the $2.5 \%$ to $97.5 \%$ percentiles (Ramachandran and Tsokos, 2021). We describe and visualize the derived median values $\left(\tilde{x}_{\mathrm{d}}\right)$ and confidence intervals in the result section.

Finally, we calculated a factor $F$ describing the relative increase between two consecutive danger levels $(d, d+1)$ :

$F=\frac{\tilde{x}_{\mathrm{d}+1}}{\tilde{x}_{\mathrm{d}}}$.

The same approach was used for all sub-levels $s_{\mathrm{i}}$.

\subsection{Consideration of forecast core zone}

Three data sources (accident and movement points, danger-level model, instability model; marked with an * in Figure 4) consistently contained the aspect- and elevation-information for each data point. Moreover, these data were available in sufficient quantity. This allowed the data to be additionally analyzed with respect to their location in relation to the critical aspect and elevation indicated in the forecast (core zone). We considered a data point as within the core zone, if both the elevation and the aspect criteria were fulfilled (see point A in Fig. 5). We considered points partly outside the core zone, if only one criterion was fulfilled, else fully outside (point B in Fig. 5). However, for danger levels 1 (low), 4 (high) and 5 (very high), we did not calculate core-zone specific values as normally no core zone is indicated at 1 (low), and as frequently all aspects and a low elevation threshold were indicated at the two highest danger levels, leaving very few data points for analysis. 
(a)

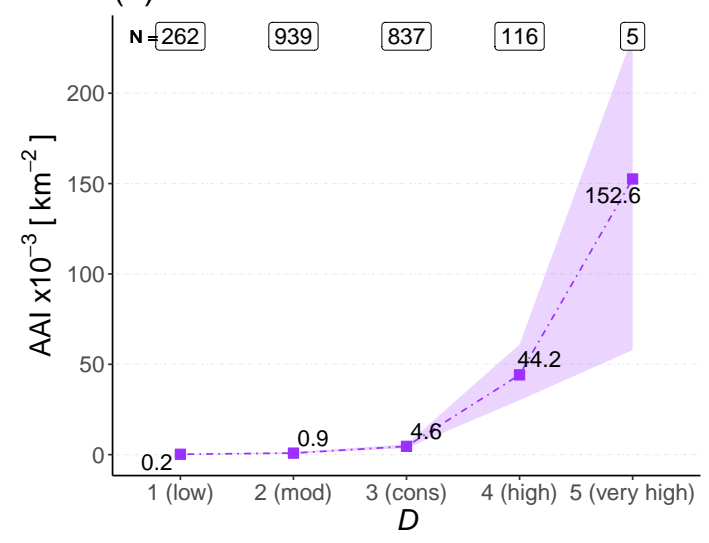

(b)

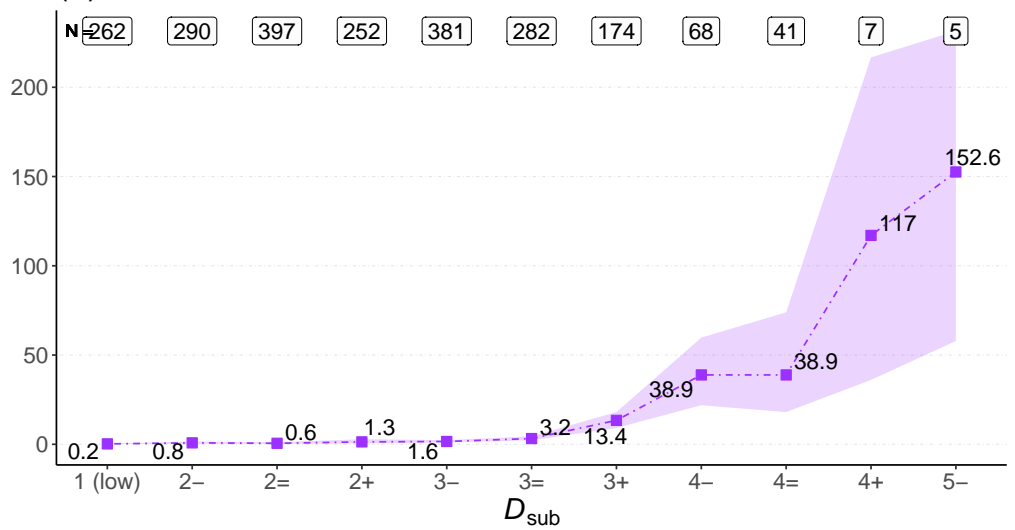

Figure 6. Avalanche activity index $(A A I)$ for natural avalanches per $1000 \mathrm{~km}^{2}$ potential release area $\left(A_{\text {PRA }}\right)$ for (a) each danger level and (b) each sub-level. $N$ represents the number of cases. Shown are the median values (points) and the $95 \%$ confidence interval (shaded area).

The entire analysis was performed using the software $R$ (R Core Team, 2020).

\section{Results}

\subsection{Natural avalanches}

Natural avalanche activity increased with increasing danger level (Fig. 6a, Tab. 2). Between 2 (moderate) and 5 (very high), the increase in the avalanche activity index $A A I$ was strong and significant between neighbouring danger level pairs (factor $F>$ $3.5, p<0.02$ ). The increase was strongest between 3 (considerable) and 4 (high) $(F=9.6$ ), and between 2 (moderate) and 3 (considerable) $(F=5.3)$. The increase between 1 (low) and 2 (moderate) was by $F=3.8(p=0.1)$. This positive correlation was also reflected in the generally continuous increase of the number of avalanches of a certain size per $1000 \mathrm{~km}^{2}\left(\tilde{\rho}_{\text {nat,i }}\right)$ with increasing $D$ (Tab. 2). On average more than one natural size 2 avalanche was reported at 1 (low) $\left(\tilde{\rho}_{\text {nat,i }} \geq 1.3\right.$ ), this threshold was only attained for size 3 avalanches at 3 (considerable) ( $\tilde{\rho}_{\text {nat, } \mathrm{i}} \geq 2.3$ ) and for avalanches of size class $\geq 4$ at 4 (high) $\left(\tilde{\rho}_{\text {nat, } \mathrm{i}} \geq\right.$ 2.7).

The increasing frequency of natural avalanche occurrence of increasing size with increasing danger level, as seen for the danger levels $D$ in Figure 6a, is well reflected in $D_{\text {sub }}$ (Fig. 6b). A significant positive correlation between $D_{\text {sub }}$ and the avalanche activity index was found $\left(r_{\mathrm{s}}=0.35, p<0.001\right)$. Exceptions to this overall steady increase in $A \tilde{A} I$ with increasing $D_{\text {sub }}$ were found between $2^{-}$and $2^{=}(F=0.7)$, and between $4^{-}$and $4^{=}(F=1.0)$. Overall, $A \tilde{A} I$ was rather low between 1 (low) and $3^{-}(A \tilde{A} I \leq 1.6)$ showing only a comparably small relative increase by a factor of 7.2 (Fig. 6b). For each danger level, an increase in $A \tilde{A} I$ between the respective sub-level minus and plus was observed. This increase was lowest between $2^{-}$and $2^{+}(F=1.7)$, and most pronounced between $3^{-}$and $3^{+}$with a factor of 8.5. Even though $A \tilde{A} I$ was higher at $5^{-}$ $(A \tilde{A} I=152.6)$ compared to $4^{+}(A \tilde{A} I=117)$, this finding is based on a very small number of samples only $(N=5$ and $N=$ 
Table 2. Spatial density of natural avalanches $\tilde{\rho}_{\text {nat,i }}$ (or number of avalanches) of size $i$ per $1000 \mathrm{~km}^{2}$ for each of the five danger levels $D$. Median values are shown.

\begin{tabular}{lccccc}
\hline & \multicolumn{5}{c}{$D$} \\
avalanche size & 1 (low) & 2 (moderate) & 3 (considerable) & 4 (high) & 5 (very high) \\
\hline 2 & 1.3 & 2.0 & 6.8 & 26.8 & 31.9 \\
3 & 0.1 & 0.5 & 2.3 & 14.3 & 24.2 \\
$\geq 4$ & 0 & 0.02 & 0.2 & 2.7 & 12.5 \\
\hline
\end{tabular}

Table 3. Spatial density of natural avalanches $\tilde{\rho}_{\text {nat,i }}$ (or number of avalanches) of size $i$ per $1000 \mathrm{~km}^{2}$ for each of the sub-levels $D_{\text {sub }}$. Median values are shown.

\begin{tabular}{lccccccccccc}
\hline & & \multicolumn{1}{c}{$D_{\text {sub }}$} & & & & & \\
avalanche size & 1 (low) & $2-$ & $2=$ & $2+$ & $3-$ & $3=$ & $3+$ & $4-$ & $4=$ & $4+$ & $5-$ \\
\hline 2 & 1.3 & 1.2 & 2.0 & 2.9 & 4.2 & 5.5 & 14.3 & 27.4 & 16.1 & 84 & 31.9 \\
3 & 0.1 & 0.5 & 0.3 & 0.6 & 0.8 & 1.9 & 5.9 & 15.2 & 8.2 & 41.2 & 24.2 \\
$\geq 4$ & 0 & 0.02 & 0 & 0.05 & 0.03 & 0.08 & 0.6 & 2.1 & 2.9 & 6.8 & 12.5 \\
\hline
\end{tabular}

7, respectively). The generally positive correlation between $D_{\text {sub }}$ and avalanche activity was also visible when analyzing the number of avalanches of a certain size class: For instance, the number of avalanches of size $\geq 4$ was very low at sub-level $\leq$ $3^{=}\left(\tilde{\rho}_{\text {nat }, \mathrm{i}} \leq 0.08\right)$, but increased continuously with increasing danger level peaking at $5^{-}\left(\tilde{\rho}_{\text {nat }, \mathrm{i}}=12.5\right)$. The number of natural avalanches of size 2 or size 3 showed the strongest increase between $2^{-}$and $4^{-}$(Tab. 3 ).

\subsection{Human-triggered avalanches and whumpfs}

\subsubsection{Human-triggered avalanches}

The number of human-triggered avalanches per $10000 \mathrm{~km}^{2}\left(\rho_{\text {hum }}\right)$ increased significantly from 1 (low) to 2 (moderate), and

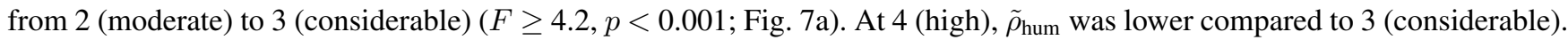
At least one human-triggered avalanche was reported on $3 \%$ of the days in regions with a forecast 1 (low) and on $50 \%$ of the days when 3 (considerable) was forecast.

At the resolution of the forecast sub-levels, the number of human-triggered avalanches $\tilde{\rho}_{\text {hum }}$ increased continuously from 1 (low) to $3^{+}(F \geq 1.2$, Fig. 7b). At 4 (high), only about half as many human-triggered avalanches were reported compared to $3^{+}$. Human-triggered avalanches were observed more than 40 times more frequently at $3^{+}$compared to 1 (low).

Human-triggered avalanches are comparably rare events. This means that $\rho_{\text {hum,i }}$ is particularly sensitive to the size of the area, as the likelihood that at least one human-triggered avalanche is reported increases with increasing potential avalanche terrain, given the same avalanche conditions. However, we were interested in true zeros (structural zeros) rather than sampling 
zeros (Ridout et al., 1998). For instance, sampling zeros may occur more often when the forecast refers to less terrain. Results obtained for approximately similar $A_{\mathrm{PRA}}$ for each danger level or sub-level showed a similar pattern, except that $\tilde{\rho}_{\text {hum }}$ peaked at $3^{=}$. The corresponding Figure A2 is shown in the Appendix.

\subsubsection{Human-triggered whumpfs and shooting cracks}

Observers seldom reported human-triggere danger signs at 1 (low), less than one in 22 observations. Danger signs were rather common at 3 (considerable) and 4 (high) when $\geq 37 \%$ of the observations indicated danger signs (Fig. 7c). These proportions increased significantly between all danger level pairs $(p<0.001, F>1.5)$. Furthermore, if danger signs were observed, an increasingly larger share was reported as frequent rather than rare with increasing danger level. For instance, $28 \%$ of the observations, which indicated danger signs, were reported as frequent at 2 (moderate), but 54\% at 4 (high).

As can be seen in Figure $7 \mathrm{~d}$, when considering $D_{\text {sub }}$, the proportions of observations mentioning danger signs increased in a strictly monotonic fashion with increasing $D_{\text {sub }}\left(F>1.1 ; r_{\mathrm{s}}=0.35, p<0.001\right)$. In addition, the proportion of reports indicating danger signs as frequent rather than rare, increased from less than $30 \%$ at $D_{\text {sub }} \leq 2^{+}$to more than $50 \%$ at 4 (high). This increase was monotonic between $2^{+}$and 4 (high). In other words, with increasing sub-level, an increasing share of observations indicated at least one danger sign, while at the same time proportionally more danger signs were observed.

\subsubsection{Accident-movement point ratio during backcountry touring}

The accident-movement point ratio ( $\left.R_{\text {acc/move }}\right)$ increased significantly from 1 (low) to 3 (considerable) $(p<0.001)$, with a relative increase by a factor of about 12 (Fig. 7e). The increase in $\tilde{R}_{\text {acc/move }}$ from 3 (considerable) $\left(\tilde{R}_{\text {acc/move }}=1.2 \times 10^{-3}\right)$ to 4 (high) $\left(\tilde{R}_{\text {acc/move }}=1.3 \times 10^{-3}\right)$ was not significant $(p=0.33)$, which is also indicated by the large confidence interval at 4 (high) $\left(\mathrm{CI}=\left[0,3.2 \times 10^{-3}\right]\right) . \tilde{R}_{\text {acc/move }}$ was significantly higher within the forecast core zone compared to fully outside the core 370 zone.

As shown in Figure 7f, $\tilde{R}_{\text {acc/move }}$ increased strictly monotonically with increasing $D_{\text {sub }}$ from 1 (low) to $3^{+}(F>1.4$ ). The total increase between 1 (low) $\left(\tilde{R}_{\text {acc/move }}=0.074 \times 10^{-3}\right)$ and $3^{+}\left(\tilde{R}_{\text {acc/move }}=2.54 \times 10^{-3}\right)$ was by a factor 33 . This increase was clearly visible also within 2 (moderate) (factor 2.5 between $2^{-}$and $2^{+}$) and 3 (considerable) (factor 2.8 between $3^{-}$and $3^{+}$). At 4 (high), the ratio was lower than at $3^{+}$, but this finding is based on very few data points ( 2 accidents, $0.2 \%$ of the movement points).

In summary, a positive monotonic relationship between data related to the frequency of locations where human triggering is possible and $D_{\text {sub }}$ exists within the range where a significant increase was noted for the conventional danger levels. 
(a)

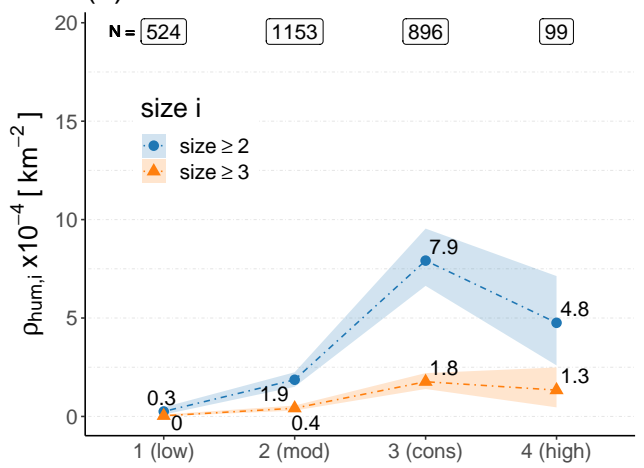

(c)

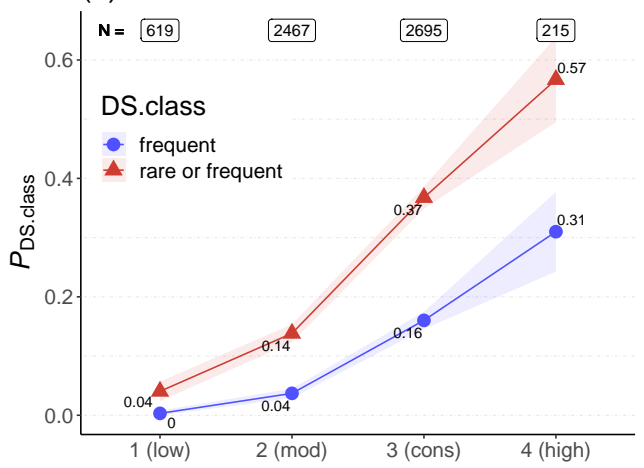

(e)

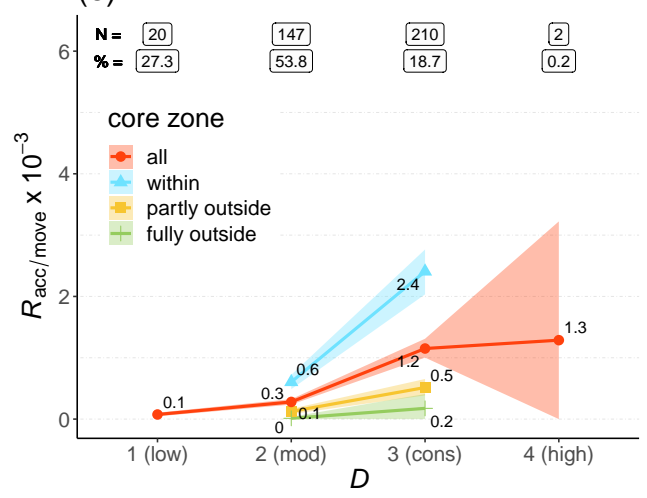

(b)

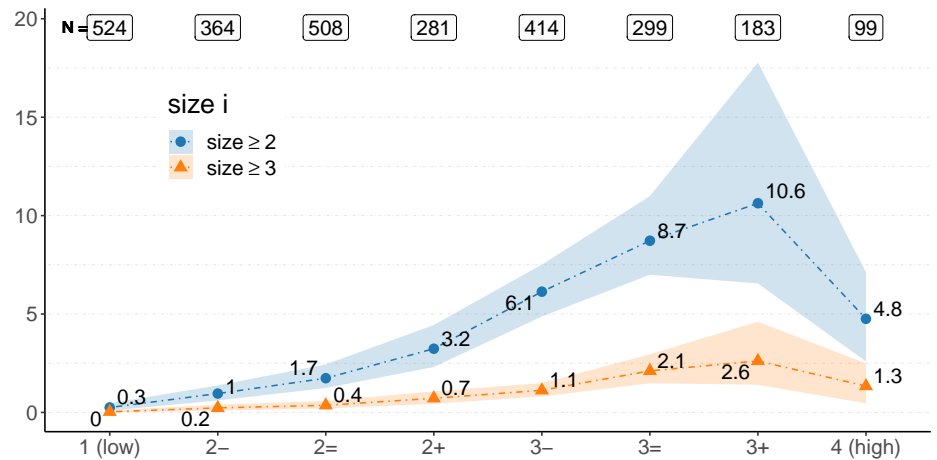

(d)

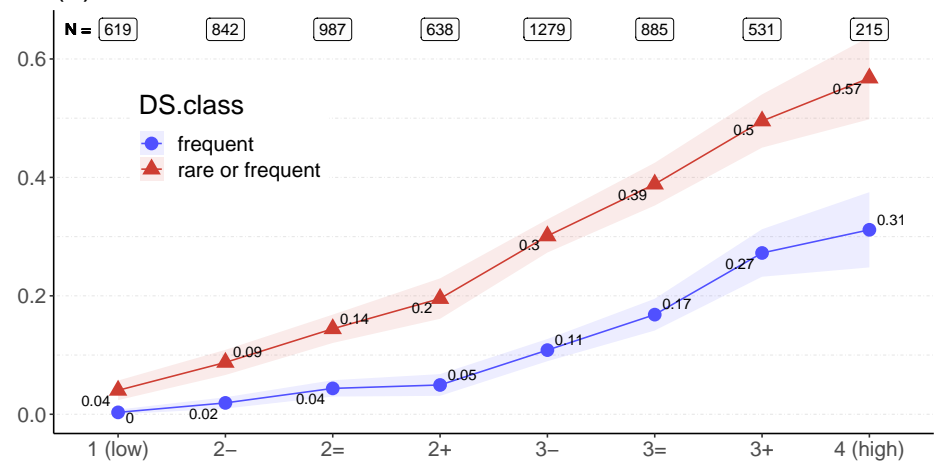

(f)

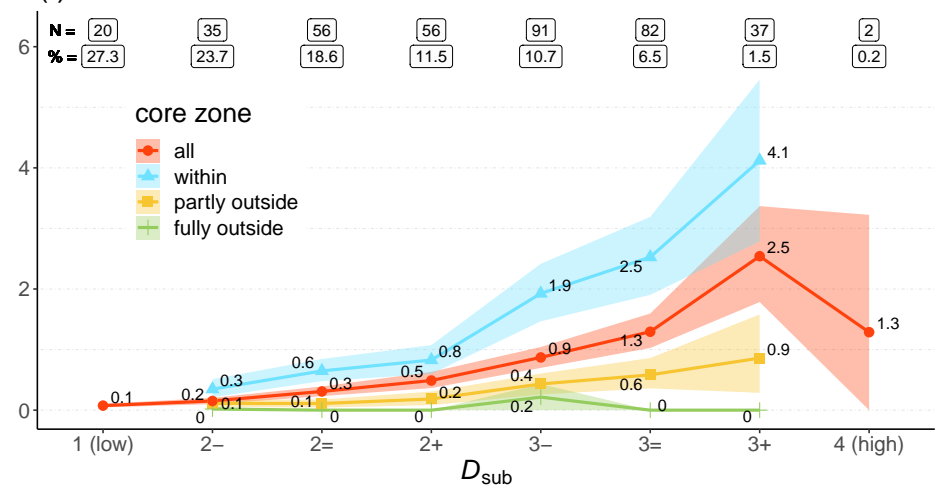

Figure 7. The density of human-triggered avalanches (or the number relative to the surface area) ( $\rho_{\text {hum,i }}$ ) (upper row), the proportion of observations with reported danger signs $\left(P_{\mathrm{DS} . c \text { lass }}\right)$ (middle row) and the ratio of accident to movement points during backcountry touring ( $R_{\text {acc/move }}$ ) (lower row) are compared to the danger level $D$ (left column) and sub-level $D_{\text {sub }}$ (right column). Shown are the median values (points) and the 95\% confidence interval (shaded area). $N$ represents the number of danger regions (upper row), the number of observations (middle row), and the number of accident points (lower row). The number of movement points is expressed as percentage (\%) relative to all movement points $N=976087$. 
(b)
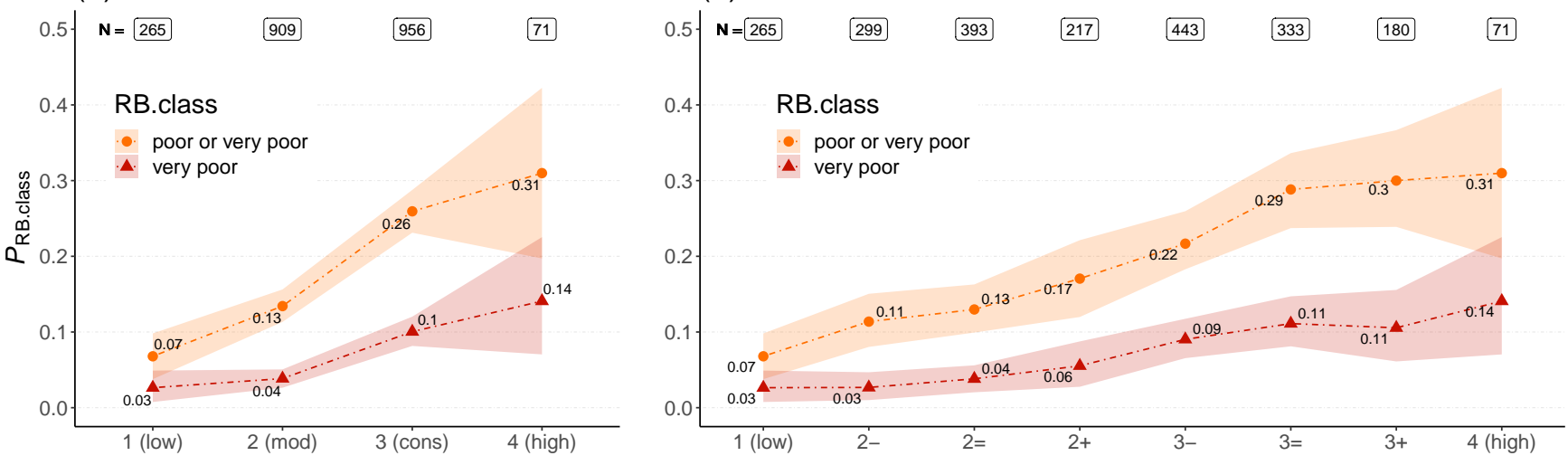

(c)
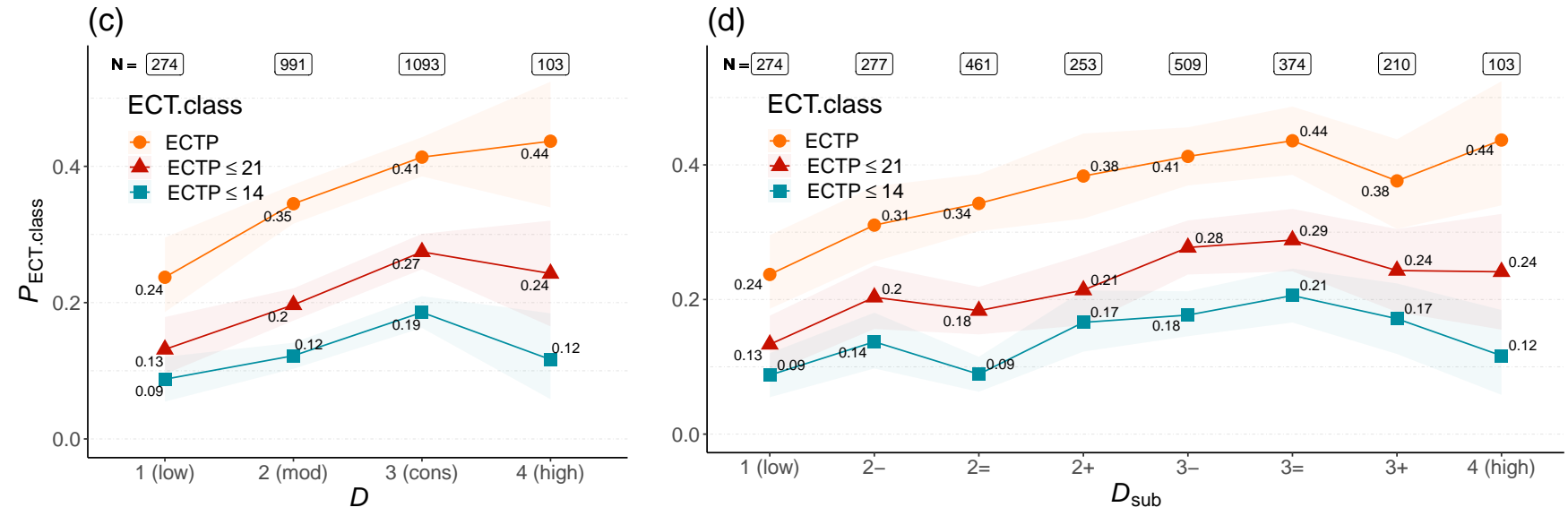

Figure 8. Proportion of Rutschblock test results ( $P_{\mathrm{RB} . c l a s s}$, upper row) and Extended Column Test results $\left(P_{\mathrm{ECT} . c l a s s}\right.$, lower row) related to instability for tests observed at a specific danger level $D$ (left column) and sub-level $D_{\text {sub }}$ (right column). Shown are the median values (points) and the $95 \%$ confidence interval (shaded area).

\subsection{Stability tests}

\subsubsection{Rutschblock test}

The median proportion of Rutschblock (RB) test results related to instability, $\tilde{P}_{\mathrm{RB} . c l a s s}$, increased with increasing danger level $D$ in a strictly monotonic fashion $\left(F>1.2\right.$, Fig. 8a). Differences in $P_{\mathrm{RB} . c l a s s}$ between danger level pairs were significant for RB.class $=$ very poor between 2 (moderate) and 3 (considerable) $(p<0.001)$, and for the combined proportion of very poor and poor test results between 1 (low) and 3 (considerable) $(p \leq 0.001)$.

Similar findings can be noted when analyzing the relationship between $D_{\text {sub }}$ and $P_{\text {RB.class }}$ (Fig. 8b): the combined proportion of very poor or poor RB test results increased continuously with increasing sub-levels ( $F \geq 1.04$ ), with a weak, but significant 
correlation $\left(r_{\mathrm{s}}=0.2, p<0.001\right)$. For RB.class $=$ very poor this increase was strictly monotonic only between $2^{-}$and $3^{=}(F \geq$ 1.2). Similarly, the correlation was weaker $\left(r_{\mathrm{s}}=0.12, p<0.001\right)$.

\subsubsection{Extended column test}

The median proportion of ECT results related to instability increased with increasing danger level from 1 (low) to 3 (considerable) ( $F>1.2$, Fig. 8c). The difference in $P_{\mathrm{ECT} . c l a s s}-$ values between subsequent danger levels was significant for $E C T P$ and $E C T P \leq 21$ from 1 (low) to 3 (considerable) $(p \leq 0.02$ ), and for $E C T P \leq 14$ between 2 (moderate) and 3 (considerable). At 4 (high), $P_{\text {ECT.class }}$-values were not significantly higher, or were even lower than at 3 (considerable).

Analyzing the correlation between $P_{\text {ECT.class }}$ and the sub-levels showed strictly increasing $\tilde{P}_{\text {ECT.class-values with increasing }}$ $D_{\text {sub }}$ between 1 (low) and $3^{=}$for ECTP. No further increase was noted at higher $D_{\text {sub }}$. Similar patterns were observed for the proportion of $E C T P \leq 21$ or $E C T P \leq 14$, although the median value slightly decreased between $2^{-}$and $2^{=}$. Again, highest

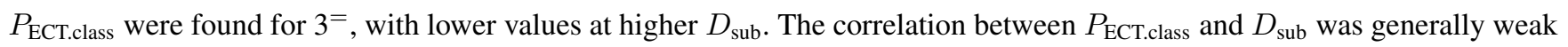
though significant $\left(r_{\mathrm{s}} \geq 0.12, p<0.001\right)$.

In summary, we observed an increasing proportion of stability tests related to instability with increasing $D_{\text {sub }}$ within the range in the danger scale where this increase was significant when comparing subsequent danger levels $D$. Similar to humantriggered avalanches (cf. Fig: 7a and b) or the accident-movement point ratio (cf. Fig. 7e and f), no further increase was noted at $3^{+}$or 4 (high).

\subsection{Models}

\subsubsection{Danger-level model}

The danger rating predicted by the danger-level model showed a strong and significant increase from 1 (low) $\left(\tilde{D}_{\text {model }}=1.44\right)$ to 4 (high) $\left(\tilde{D}_{\text {model }}=3.14, p<0.001\right.$; Fig. 9 a). The absolute increase was on average by 0.5 to 0.6 from one danger level to the next, rather than a full level. Similar significant differences were found for predictions within the forecast core zone compared to those which were at locations and for aspects which were fully outside the core zone. The difference between these predictions was by about $0.5(p<0.001)$, and thus similar to the difference between neighbouring danger levels.

Turning to $D_{\text {sub }}$, the same patterns can be noted (Fig. 9b): $\tilde{D}_{\text {model }}$ increased continuously with increasing $D_{\text {sub }}(F \geq 1.04)$. The correlation was strong and significant $\left(r_{\mathrm{s}}=0.79, p<0.001\right)$. The absolute increase from one sub-level to the next higher one was smallest from $2^{=}$to $2^{+}$(by 0.07 ), for all other pairs the increase was $\geq 0.21$. Furthermore, $\tilde{D}_{\text {model }}$ was consistently higher within the core zone compared to fully outside the core zone. 
(a)

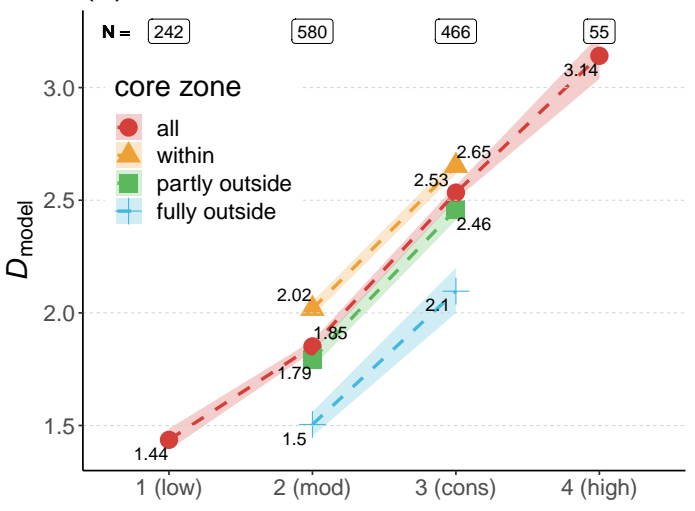

(c)

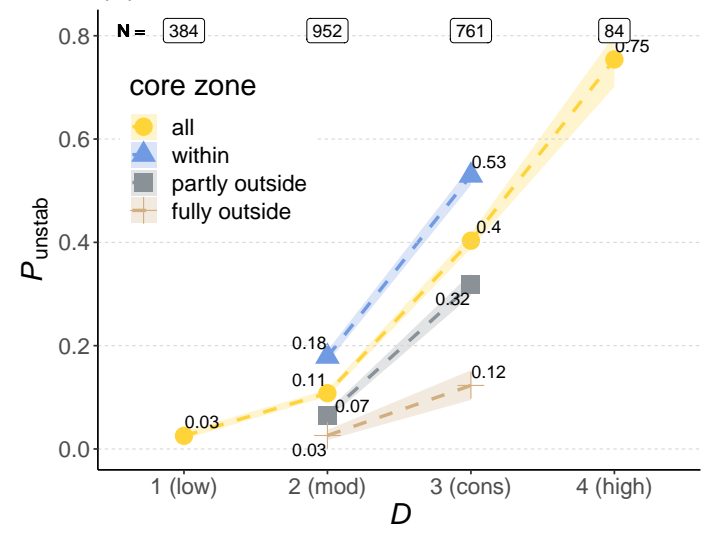

(b)

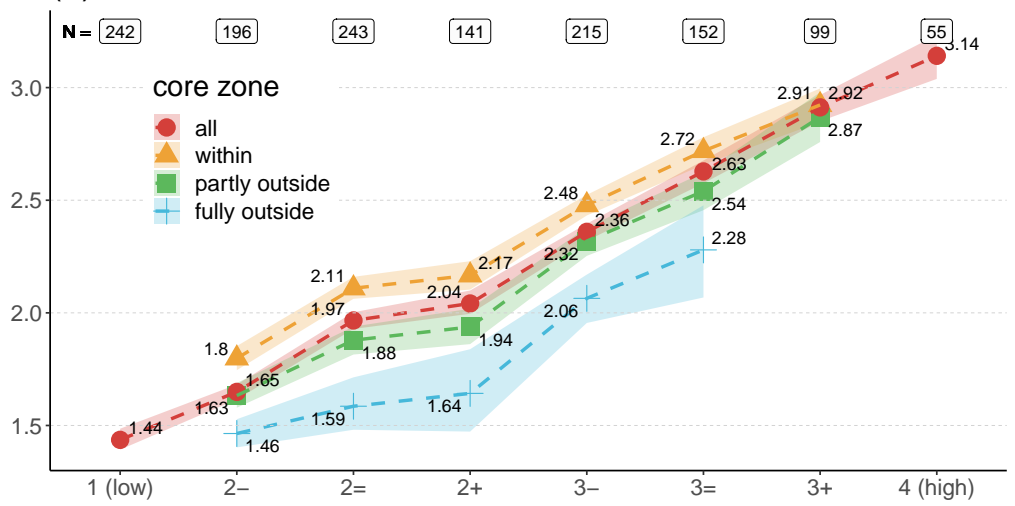

(d)

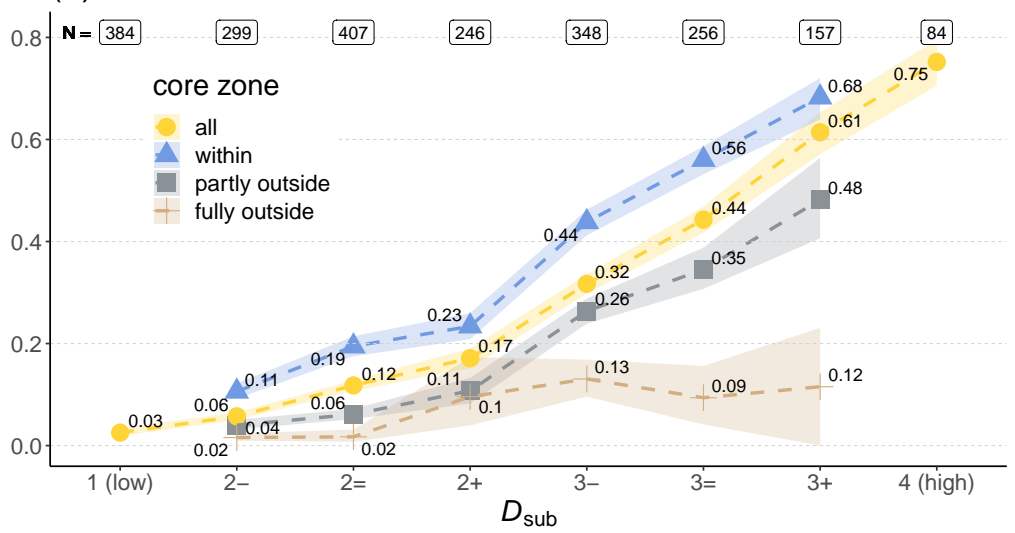

Figure 9. Output from random forest models predicting the danger level (upper row) and instability (lower row). The mean predicted danger level $\left(D_{\text {model }}\right)$ and the proportion of simulated snow profiles predicted as unstable $\left(P_{\text {unstab }}\right)$ are shown for all cases with the same danger level

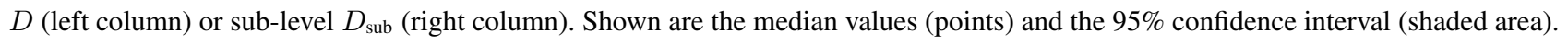

\subsubsection{Instability model}

The median proportion of simulated profiles classified as unstable $\left(\tilde{P}_{\text {unstab }}\right)$ increased significantly with increasing danger level

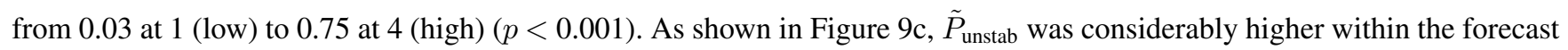
core zone than fully outside $(p<0.001)$.

Findings were similar when exploring the correlation between $P_{\text {unstab }}$ and $D_{\text {sub }}$ (Fig. 9d): $\tilde{P}_{\text {unstab }}$ increased monotonically with increasing $D_{\text {sub }}$ showing a strong, positive correlation $\left(r_{\mathrm{s}}=0.76, p<0.001\right)$. In addition, values within the core zone were always higher than outside the core zone. It is further noteworthy that $\tilde{P}_{\text {unstab-values were similarly low outside the core }}$ zone for all sub-levels within 3 (considerable) $\left(\tilde{P}_{\text {unstab }} \leq 0.13\right)$. 
Table 4. Table summarizing whether an increase (light blue, $F>1.05$ ) or a decrease (light orange, $F<0.95$ ) in the median was observed from one sub-level $\left(s_{\mathrm{i}}\right)$ to the next higher one $\left(s_{\mathrm{i}+1}\right)$. The dashed arrows indicate the range, for which significant increases between neighbouring danger level pairs $(d, d+1)$ were observed, and where, thus, an increase between sub-levels can be expected if their relative assignment would on average be correct.

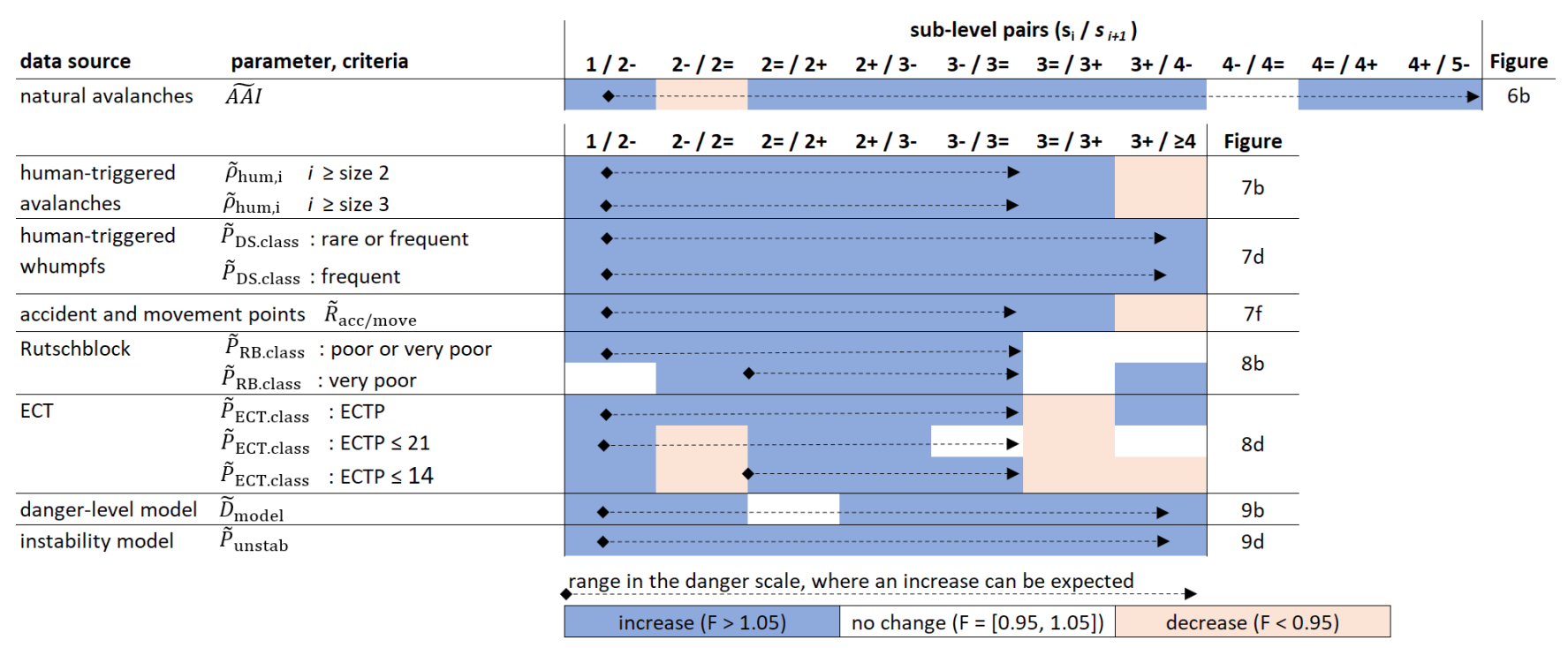

\section{Discussion}

The overarching research question we explored was: Given the daily observations and measurements, often still incomplete at the time when avalanche forecasters in Switzerland meet for their afternoon forecaster briefing, and a numerical weather prediction model, can human avalanche forecasters forecast avalanche hazard for the following day with higher resolution than the five danger levels? To this end, we analyzed a wide variety of data related to the contributing factors of avalanche hazard and investigated their relationship with sub-levels assigned to danger levels in Switzerland. The specific question we had was therefore: Given the current forecasting set up in Switzerland, are the sub-levels assigned in a way that they express the expected rank-order relationship between the three contributing factors of avalanche hazard and the sub-levels? As we could not rely on a clear definition of the sub-levels, we split the analysis into two steps: first, we determined the range of the danger scale in which a given data source was valuable to distinguish between danger levels. And second, we analyzed whether a monotonic correlation between sub-levels and the data source existed.

First (RQ1), we determined in which range of the danger scale a data source was suitable for our analysis. As summarized in Table 4 by the arrows, natural avalanches, human-triggered whumpfs, and the two models were the most suitable, allowing the analysis of the entire range of the danger scale for natural avalanches, and from 1 (low) to 4 (high) for the other three data sources. In contrast, and except for the human-triggered whumpfs, data that requires a human being present in avalanche terrain was most suitable at danger levels 1 (low) to 3 (considerable). Of limited use were the two stability tests, and here particularly 
the stability classes with the most restrictive class thresholds $\left(P_{\mathrm{RB} . c l a s s}=\right.$ very poor, $\left.P_{\mathrm{ECT} . c l a s s}=\mathrm{ECTP} \leq 14\right)$. This first step was not only an important foundation for the second part of our analysis, it also confirmed that - on average - the forecast danger levels have the intended predictive value concerning the three contributing factors of avalanche hazard.

Turning to our main research question (RQ2), we summarize an increase in the value of the analyzed parameters for most of the sub-level pairs $\left(s_{\mathrm{i}}, s_{\mathrm{i}+1}\right)$, within the range where this could be expected if the relative assignment of the sub-levels was consistent on average and if the data permitted this (RQ1). Of the 74 sub-level pair comparisons shown in Table 4, 69 showed an increase from $s_{\mathrm{i}}$ to $s_{\mathrm{i}+1}$ with $F \geq 1.05$ (light blue cells), and only two a decrease ( $F \leq 0.95$, light orange cells).

These findings represent the average. It is clear, however, that there will be errors in both the forecast danger level (absolute judgment) and the forecast sub-level (comparative judgment). For instance, in a recent study exploring the agreement between local nowcast danger level assessments after a day in the field and the forecast regional danger level, 19\% of the cases when two local nowcasts in the same small warning region unanimously indicated the same danger level were different than the forecast danger level Techel et al. (2020b). However, in these cases, the difference was often less than a full danger level: most often (70\%) the sub-level qualifier was the one closest to the local estimates, a fact not known to the local observers. This highlights that assigning a sub-level can provide an important indication about the tendency within a danger level, and thus has the potential to reduce the magnitude of the forecast error. A useful example to illustrate this is the comparison of natural avalanche activity between neighbouring sub-levels belonging to two danger levels, as for instance, $4^{+}$and $5^{-}$. The avalanche activity was more similar at these two sub-levels $(A \tilde{A} I=117$ and $A \tilde{A} I=153$, respectively, Fig. $6 \mathrm{~b})$ than when comparing 4 (high) $(A \tilde{A} I=44)$ with 5 (very high) $(A \tilde{A} I=153$, Fig. 6a).

\subsection{Implications for forecasters}

Our analysis showed that forecasters can estimate sub-levels based on the available data, thus providing a way of increasing the resolution of the forecast danger level, while maintaining the well-established standard of assessing and communicating avalanche hazard using the five danger levels. Moreover, the comparison with the two models not used in the forecast production process indicated that the sub-level forecasts were reasonably consistent. The models mirrored differences in the forecast danger level and the sub-level, as well as concerning aspects and elevations where the danger prevailed.

Refined danger ratings allow to express a more natural and gradual change of avalanche danger compared to the five danger levels. While models have the potential to provide continuous output, such an approach is not possible for humans. Therefore, the experts assessed avalanche danger in two stages combining an absolute and a relative judgment (Kahneman et al., 2021): First, forecasters determined the danger level before they performed a comparative sorting within this level. The definition of the danger levels provides the absolute anchor, while the forecasters' experience concerning the variation within a danger level is relevant for the comparative judgment. Based on our findings, we conclude that the specification of a sub-level is possible using such a procedure, regardless of whether an avalanche warning service relies on measurements, observations, and a weather forecast; or whether the forecast production relies more strongly on numerical models. However, prerequisites to refine sub-levels are that data relevant to the forecasting task is sufficiently available in time and space, and that the assessment is made using a sufficiently detailed spatial and temporal resolution (Techel et al., 2020b). In conventional avalanche hazard 
assessment, increasing the resolution of the avalanche forecast is limited by the data available at the time of the assessment and the available resources of the avalanche forecasters. With the use of models, the resolution can be increased and at the same time the noise, i.e. the random errors, can be reduced. Thus, in the future, such models could provide a viable addition to assess and forecast avalanche danger at a regional level and with greater spatial and temporal resolution, complementary to the more conventional way of forecasting.

While we have shown that the method of combining absolute and relative judgments can result in avalanche danger assessments with finer granularity, it might still be advantageous to describe typical characteristics for each sub-level. This may not only help forecasters when deciding on a sub-level but may potentially also be useful for users of this information. Therefore, we envision that using the presented data, but also the actual descriptions of avalanche danger in the avalanche forecast (Hutter et al., 2021), a data-driven description of the sub-levels could be obtained.

\subsection{Practical applications}

We have demonstrated that, on average, the forecast sub-levels have predictive value, that is, they correlate with the three contributing factors of avalanche hazard. Therefore, we argue, the sub-levels should be provided in a suitable form to forecast users, as they may support the decision-making process.

We see two potential use cases: The first, more traditional use case, is the provision of the sub-levels as part of the avalanche forecast product, permitting a direct interpretation of the sub-level by the human forecast user. However, as several studies have shown, the comprehension of the information communicated in the bulletin is strongly related to the education of the user, and to the complexity of the avalanche situation (e.g. Engeset et al., 2018; St. Clair et al., 2021). Therefore, we consider it important that the provision of this information to the public does not violate the structure of the information pyramid. This can be taken into account by retaining the defined danger levels and their (optional) subdivision (sub-level). Questions that arise are, for instance, for which user group this additional information should be available, and how it should be presented as not to reduce comprehensibility of the forecast. Another option would be to pass on this information to the public indirectly, by feeding it primarily into algorithms, which build upon the avalanche forecast, such as a classification of avalanche risk on ski tours as on the website www.skitourenguru.ch (Schmudlach, 2022). When used by such algorithms, sub-levels can increase the precision of the forecast without causing problems with comprehensibility.

Second, the sub-levels could also be used for the development and validation of models. These may, in turn, improve avalanche forecasting. One such example is the danger-level model, which was trained and validated with the defined danger levels (Pérez-Guillén et al., 2021). The danger-level model already captured differences in avalanche danger between the sub-levels and the core zone. However, we surmise that re-training the model incorporating the information contained in the sub-level may potentially increase the model performance further.

\subsection{Limitations}

505 We aimed at exploring the correlation between $D_{\text {sub }}$ and data related to the contributing factors of avalanche hazard. However, the results are not only influenced by the quality of $D_{\text {sub }}$, but also by potential errors in the assignment of a danger level 
$D$, which is the first step in the assessment process, or in the spatial clustering of warning regions to regions with the same conditions (cf. danger regions shown in Fig. 2a). In addition to errors related to the forecast, errors and bias may also be present in the data used in this analysis. Of particular relevance are non-random errors or bias, for instance, due to sampling or reporting preferences, or due to human behaviour as a consequence of avalanche conditions. As we can't decompose the analysis into these various error sources, we are unable to quantify these. However, assuming that non-random errors or the magnitude of bias in the data do not change abruptly between consecutive sub-levels, we argue that overall trends should be captured.

Our study was set in Switzerland. While the results can therefore not readily be applied to other countries, we believe that the more general finding, namely the approach of combining absolute and relative judgments, should be applicable in other forecast settings as well.

The distribution of the data was not uniform over the entire forecast domain. For instance, hardly any data was available for the Jura or the middle and southern Ticino (region B in Figure 2a). Thus, it is unclear whether the assignment of the sub-levels is of equal quality in these areas. Furthermore, for the higher danger levels and sub-levels (4 (high) and 5 (very high)), the data sets are comparably small.

\section{Conclusions}

Can forecasts of avalanche danger be refined by using a combination of absolute and comparative judgments? We addressed this question by comparing five years of Swiss avalanche forecasts including a sub-level qualifier (comparative judgment) assigned to the danger level (absolute judgment) with several data sources considered a proxy for the three contributing factors of avalanche hazard. We have shown that, on average, these sub-levels reflect the expected increase in the number of locations with poor snow stability and in the number and size of avalanches with increasing forecast sub-level.

Our findings are specific to the current forecast set up in Switzerland. However, we surmise that avalanche warning services whose hazard assessment is based on a similar temporal and spatial scale as is used in Switzerland should also be able to refine their assessments if (1) relevant data in time and space is available, and (2) if a similar approach combining absolute and relative judgments is used.

530 The sub-levels clearly increase the predictive value of the forecast, opening the discussion on how this information could be provided to forecast users.

Data availability. The data collected as part of operational avalanche forecasting will be made available at the data repository www.envidat. ch. 
(a)

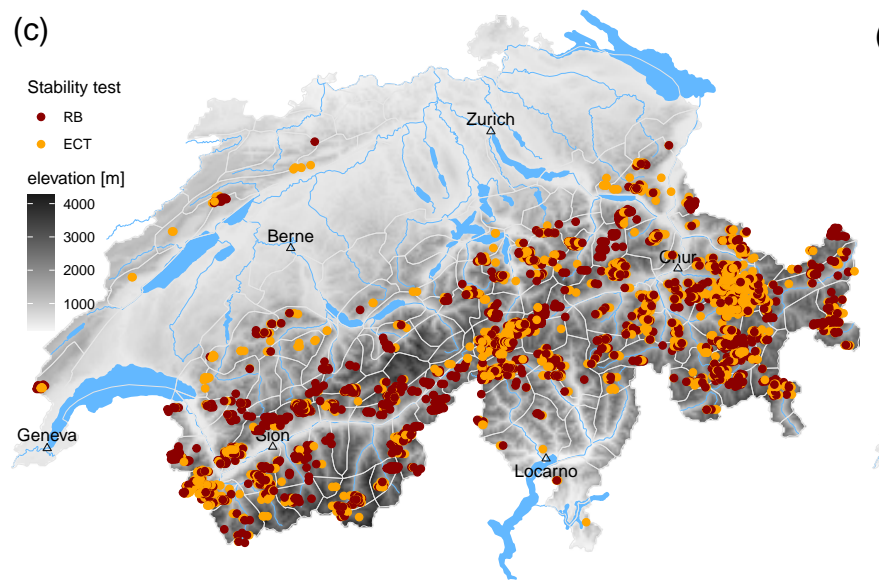

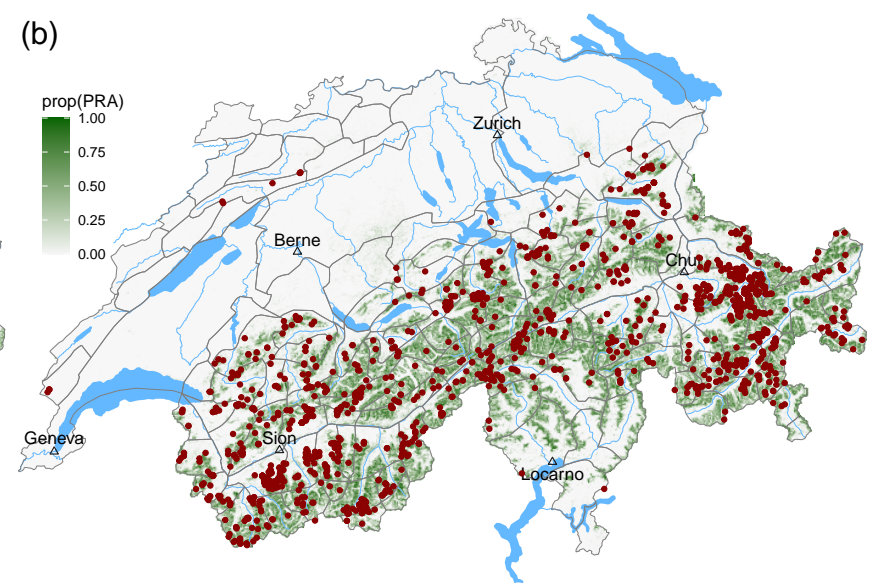

(b)

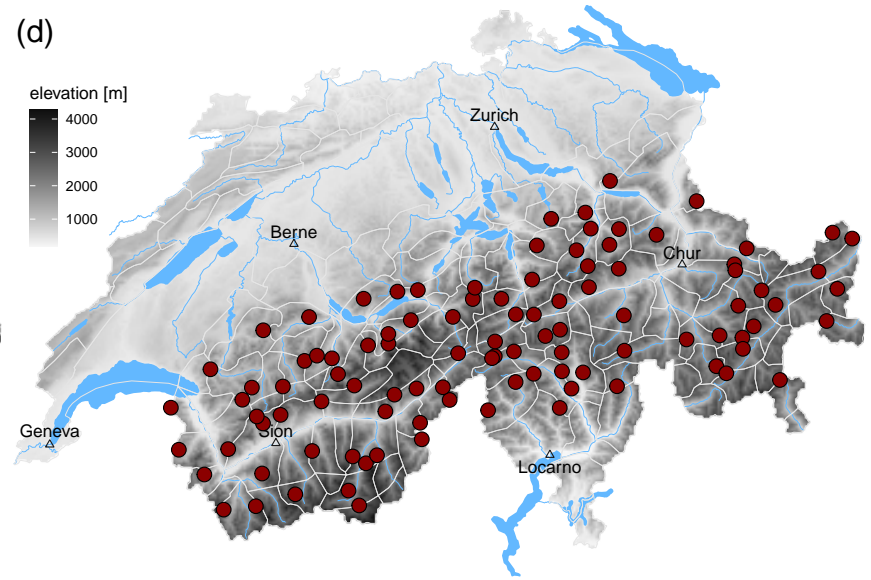

Figure A1. Maps of Switzerland showing (a) the warning regions (grey polygon boundaries) and those selected for the analysis of natural avalanches (bold polygons), (b) the location of human-triggered avalanches (dots), (c) the location of stability tests (dots), and (d) the location of the automatic weather stations, where the two models were run (points). For illustration purposes, colour shading in the background represents (a, b) the proportion of potential release areas (prop(PRA)) according to Bühler et al. (2018) per $500 \times 500 \mathrm{~m}$ grid cells, and (c, d) elevation based on a digitial elevation model (Source: SwissTopo).

Author contributions. Study design: FT, KW; data curation and extraction: FT, GS; analysis: FT; models: SM, CP; manuscript writing: FT; manuscript reviewing: FT, SM, CP, GS, KW.

Competing interests. Two of the authors (FT, KW) are avalanche forecasters, directly involved in the production of the forecast. 
(a)

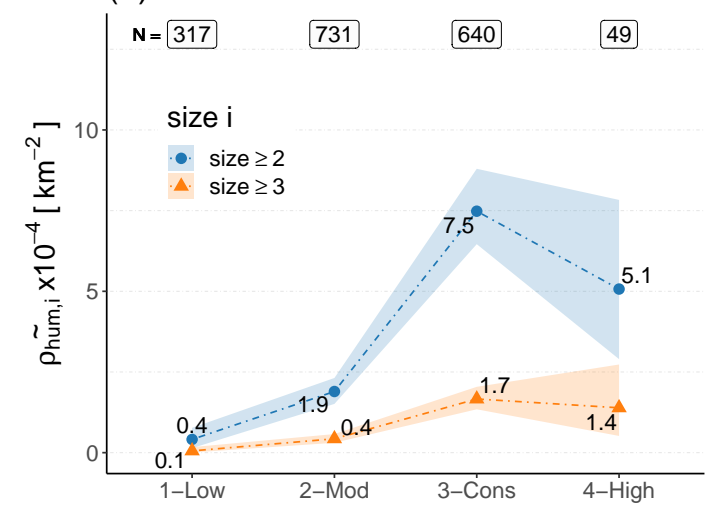

(b)

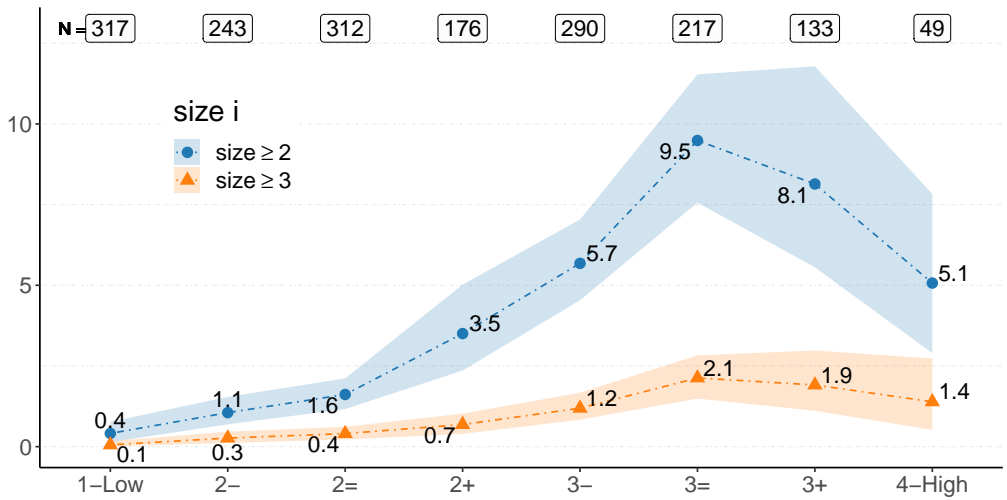

Figure A2. The density of human-triggered avalanches (or the number relative to the surface area) compared to (a) the danger level $D$ and

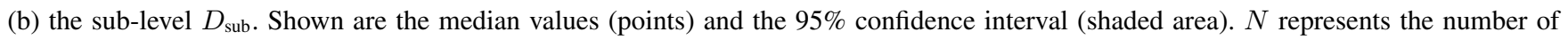
danger regions. Here, we restricted the analysis to cases, when the same $D_{\text {sub }}$ was forecast for regions with approximately similar $A_{\mathrm{PRA}}$. The resulting median $A_{\text {PRA }}$ was between 2000 and $2300 \mathrm{~km}^{2}$ for each $D_{\text {sub }}$.

\section{References}

Birkeland, K.: Spatial patterns of snow stability through a small mountain range, Journal of Glaciology, 47, 176-186, https://doi.org/10.3189/172756501781832250, 2001.

Breiman, L.: Random forests, Machine Learning, 45, 5-32, https://doi.org/10.1023/A:1010933404324, 2001.

Bühler, Y., von Rickenbach, D., Stoffel, A., Margreth, S., Stoffel, L., and Christen, M.: Automated snow avalanche release area delineation validation of existing algorithms and proposition of a new object-based approach for large-scale hazard indication mapping, Natural Hazards and Earth System Sciences, 18, 3235-3251, https://doi.org/10.5194/nhess-18-3235-2018, https://www.nat-hazards-earth-syst-sci.net/ 18/3235/2018/, 2018.

EAWS: European Avalanche Danger Scale (2018/19), https://www.avalanches.org/wp-content/uploads/2019/05/European_Avalanche_ Danger_Scale-EAWS.pdf, last access: 14 Feb 2020, 2018.

EAWS: Standards: avalanche size, https://www.avalanches.org/standards/avalanche-size/, last access: 09/09/2019, 2019.

550 EAWS: Standards: Avalanche danger scale, https://www.avalanches.org/standards/avalanche-danger-scale/, last access: 2020/11/03, 2020.

EAWS: Definition of avalanche danger, avalanche danger level and their contributing factors; presented at EAWS General Assembly, Davos, Switzerland, 2021, EAWS working group Matrix and Scale (working group members: Müller, K.; Bellido, G.; Bertrando, L.; Feistl, T.; Mitterer, C.; Palmgren, P.; Sofia, S.; Techel, F.). presented at: EAWS General Assembly, Davos, Switzerland, June $2021,2021$.

Efron, B.: Bootstrap methods: another look at the jackknife, Annals of Statistics, 7, 1-26, 1979.

Engeset, R. V., Pfuhl, G., Landrø, M., Mannberg, A., and Hetland, A.: Communicating public avalanche warnings - what works?, Nat Hazards Earth Syst Sci, 18, 2537-2559, https://doi.org/10.5194/nhess-2018-183, 2018.

Floyer, J., Klassen, K., Horton, S., and Haegeli, P.: Looking to the 20's: computer-assisted avalanche forecasting in Canada, in: Proceedings ISSW 2016. International Snow Science Workshop, 2-7 October 2016, Breckenridge, Co., pp. 1245-1249, 2016. 
Goffin, R. and Olson, J.: Is it all relative? Comparative judgments and the possible improvement of self-ratings and ratings of others,

Perspectives on Psychological Science, 6, 48-60, https://www.jstor.org/stable/41613423, 2011.

Hollander, M. and Wolfe, D.: Nonparametric Statistical Methods, New York: John Wiley and Sons, 1973.

Hutter, V., Techel, F., and Purves, R. S.: How is avalanche danger described in textual descriptions in avalanche forecasts in Switzerland? Consistency between forecasters and avalanche danger, Natural Hazards and Earth System Sciences, 21, 3879-3897, https://doi.org/10.5194/nhess-2021-160, 2021.

565 Kahneman, D., Sibony, O., and Sunstein, C.: Noise: A flaw in human judgment, William Collins, London, U.K., 2021.

Kuter, K.: Essential probability theory for data science (DSCI 500B), Saint Mary's College, https://stats.libretexts.org/Courses/Saint_Mary' s_College_Notre_Dame/DSCI_500B_Essential_Probability_Theory_for_Data_Science_(Kuter), online; last access: 8 Feb $2022,2020$.

LaChapelle, E.: The fundamental process in conventional avalanche forecasting, Journal of Glaciology, 26, 75-84, https://doi.org/10.3189/S0022143000010601, 1980.

Lehning, M., Bartelt, P., Brown, B., Russi, T., Stöckli, U., and Zimmerli, M.: Snowpack model calculations for avalanche warning based upon a new network of weather and snow stations, Cold Reg. Sci. Technol., 30, 145 - 157, https://doi.org/10.1016/S0165-232X(99)00022-1, 1999.

Lehning, M., Bartelt, P., Brown, R., Fierz, C., and Satyawali, P.: A physical SNOWPACK model for the Swiss avalanche warning; Part II. Snow microstructure, Cold Reg. Sci. Technol., 35, 147-167, 2002.

Logan, S. and Greene, E.: Patterns in avalanche events and regional scale avalanche forecasts in Colorado, USA, in: Proceedings ISSW 2018. International Snow Science Workshop, 7 - 12 Oct 2018, Innsbruck, Austria, pp. 1059-1062, 2018.

MacGregor, D.: Principles of forecasting, vol. 30 of International Series in Operations Research Management Science, chap. Decomposition for judgmental forecasting and estimation, pp. 107-123, Springer, Boston, MA, https://doi.org/10.1007/978-0-306-47630-3_6, 2001.

Mayer, S., Herwijnen, A., Techel, F., and Schweizer, J.: A random forest model to assess snow instability from simulated snow stratigraphy, The Cryosphere Discussions, [submitted], 2022.

McClung, D.: The elements of applied avalanche forecasting, part I: The human issues, Natural Hazards, 26, 111-129, https://doi.org/10.1023/A:1015665432221, 2002.

McClung, D. and Schaerer, P.: The Avalanche Handbook, The Mountaineers, Seattle, WA., 3rd edn., 2006.

MeteoSwiss: COSMO forecasting system, https://www.meteoswiss.admin.ch/home/measurement-and-forecasting-systems/ warning-and-forecasting-systems/cosmo-forecasting-system.html, last access: 6 Jan 2022, 2022.

Miller, G.: The magical number seven, plus or minus two: Some limits on our capacity for processing information, Psychological Review, 63, 81-97, https://doi.org/10.1037/h0043158, 1956

Morin, S., Horton, S., Techel, F., Bavay, M., Coléou, C., Fierz, C., Gobiet, A., Hagenmuller, P., Lafaysse, M., Ližar, M., Mitterer, C., Monti, F., Müller, K., Olefs, M., Snook, J. S., van Herwijnen, A., and Vionnet, V.: Application of physical snowpack models in support of operational avalanche hazard forecasting: A status report on current implementations and prospects for the future, Cold Regions Science and Technology, p. 102910, https://doi.org/https://doi.org/10.1016/j.coldregions.2019.102910, 2019.

Murphy, A. H.: What is a good forecast? An essay on the nature of goodness in weather forecasting, Weather and Forecasting, 8, 281-293, https://doi.org/10.1175/1520-0434(1993)008<0281:WIAGFA>2.0.CO;2, 1993

Newcombe, R. G.: Interval estimation for the difference between independent proportions: comparison of eleven methods, Statistics in Medicine, 8, 873-890, https://doi.org/10.1002/(sici)1097-0258(19980430)17:8<873::aid-sim779>3.0.co;2-i, 1998. 
Pérez-Guillén, C., Techel, F., Hendrick, M., Volpi, M., van Herwijnen, A., Olevski, T., Obozinski, G., Pérez-Cruz, F., and Schweizer, J.:

Data-driven automated predictions of the avalanche danger level for dry-snow conditions in Switzerland, Natural Hazards Earth System Sciences Discussion, https://doi.org/10.5194/nhess-2021-341, 2021.

R Core Team: R: A Language and Environment for Statistical Computing, R Foundation for Statistical Computing, Vienna, Austria, https: //www.R-project.org/, 2020.

Ramachandran, K. M. and Tsokos, C. P.: Mathematical Statistics with Applications in R, chap. Chapter 13 - Empirical methods, pp. 531-568, Academic Press, 3rd edn., https://doi.org/10.1016/B978-0-12-817815-7.00013-0, 2021.

Ridout, M., Demetrio, C., and Hinde, J.: Models for count data with many zeros, in: International Biometric Conference, Cape Town, Dec 1998, p. 13, https://www.semanticscholar.org/paper/Models-for-count-data-with-many-zeros-Ridout-Dem\%C3\%A9trio/ 6a99f29a84a90284dabc3396296ab6cea806aa37, 1998.

Schmudlach, G.: Avalanche Risk Property Dataset (ARPD), https://info.skitourenguru.ch/download/data/ARPD_Manual_3.0.13.pdf, (data set), 2021 .

Schmudlach, G.: Skitourenguru, https://www.skitourenguru.ch, last access: 6 Jan 2022, 2022.

Schweizer, J.: The Rutschblock test - procedure and application in Switzerland, The Avalanche Review, 20, 14-15, 2002.

610 Schweizer, J. and Jamieson, B.: Snowpack tests for assessing snow-slope instability, Annals of Glaciology, 51, 187-194, https://doi.org/10.3189/172756410791386652, 2010.

Schweizer, J., Kronholm, K., and Wiesinger, T.: Verification of regional snowpack stability and avalanche danger, Cold Reg. Sci. Technol., 37, 277-288, https://doi.org/10.1016/S0165-232X(03)00070-3, 2003.

Schweizer, J., Mitterer, C., Techel, F., Stoffel, A., and Reuter, B.: On the relation between avalanche occurrence and avalanche danger level,

The Cryosphere, https://doi.org/10.5194/tc-2019-218, 2020.

Schweizer, J., Mitterer, C., Reuter, B., and Techel, F.: Avalanche danger level characteristics from field observations of snow instability, The Cryosphere, 15, 3293-3315, https://doi.org/10.5194/tc-15-3293-2021, 2021.

Simenhois, R. and Birkeland, K.: The Extended Column Test: Test effectiveness, spatial variability, and comparison with the Propagation Saw Test, Cold Regions Science and Technology, 59, 210-216, https://doi.org/10.1016/j.coldregions.2009.04.001, 2009.

SLF: Avalanche bulletin interpretation guide, WSL Institute for Snow and Avalanche Research SLF, http://www.slf.ch/lawineninfo/ zusatzinfos/interpretationshilfe/interpretationshilfe_e.pdf, edition December 2020, 53p., 2020.

SLF: SLF-Beobachterhandbuch (observational guidelines), 55 p., 2020.

SLF: Description of automated stations, https://www.slf.ch/en/avalanche-bulletin-and-snow-situation/measured-values/ description-of-automated-stations.html, last access: 6 Jan 2022, 2022.

St. Clair, A., Finn, H., and Hageli, P.: Where the rubber of the RISP model meets the road: Contextualizing risk information seeking and processing with an avalanche bulletin user typology, International Journal of Disaster Risk Reduction, 66, 102626, https://doi.org/10.1016/j.ijdrr.2021.102626, 2021.

Statham, G., Haegeli, P., Greene, E., Birkeland, K., Israelson, C., Tremper, B., Stethem, C., McMahon, B., White, B., and Kelly, J.: A conceptual model of avalanche hazard, Natural Hazards, 90, 663 - 691, https://doi.org/10.1007/s11069-017-3070-5, 2018a.

630 Statham, G., Holeczi, S., and Shandro, B.: Consistency and accuracy of public avalanche forecasts in Western Canada, in: Proceedings ISSW 2018. International Snow Science Workshop, 7 - 12 Oct 2018, Innsbruck, Austria., pp. 1491 - 1496, 2018 b.

Techel, F. and Schweizer, J.: On using local avalanche danger level estimates for regional forecast verification, Cold Regions Science and Technology, 144, 52 - 62, https://doi.org/10.1016/j.coldregions.2017.07.012, 2017. 
https://doi.org/10.5194/nhess-2022-54

Preprint. Discussion started: 23 February 2022

(c) Author(s) 2022. CC BY 4.0 License.

(c) (1)

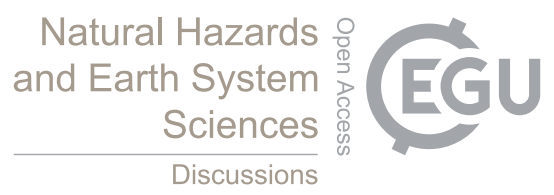

Techel, F., Müller, K., and Schweizer, J.: On the importance of snowpack stability, the frequency distribution of snowpack stability and avalanche size in assessing the avalanche danger level, The Cryosphere, 14, 3503 - 3521, https://doi.org/10.5194/tc-2020-42, 2020a.

Techel, F., Pielmeier, C., and Winkler, K.: Refined dry-snow avalanche danger ratings in regional avalanche forecasts: consistent? And better than random?, Cold Regions Science and Technology, 180, 103 162, https://doi.org/10.1016/j.coldregions.2020.103162, 2020 b.

Techel, F., Winkler, K., Walcher, M., van Herwijnen, A., and Schweizer, J.: On snow stability interpretation of extended column test results, Natural Hazards Earth System Sciences, 20, 1941-1953, https://doi.org/10.5194/nhess-2020-50, 2020c.

640 Walcher, M., Mitterer, C., and Lanzanasto, N.: A concept of harmonizing regional avalanche forecasting, in: Proceedings International Snow Science Workshop ISSW, Innsbruck, Austria, pp. 1166 - 1171, 2018.

Wilks, D.: Statistical methods in the atmospheric sciences, vol. 100 of International Geophysics Series, Academic Press, San Diego CA, U.S.A, 3rd edn., 2011.

Winkler, K. and Schweizer, J.: Comparison of snow stability tests: Extended Column Test, Rutschblock test and Compression Test, Cold Regions Science and Technology, 59, 217-226, https://doi.org/10.1016/j.coldregions.2009.05.003, 2009.

Winkler, K., Schmudlach, G., Degraeuwe, B., and Techel, F.: On the correlation between the forecast avalanche danger and avalanche risk taken by backcountry skiers in Switzerland, Cold Regions Science and Technology, 188, 103 299, https://doi.org/https://doi.org/10.1016/j.coldregions.2021.103299, https://www.sciencedirect.com/science/article/pii/ S0165232X2100080X, 2021. 